\title{
Bypassing UGC from Some Optimal Geometric Inapproximability Results
}

\author{
VENKATESAN GURUSWAMI, Carnegie Mellon University \\ PRASAD RAGHAVENDRA, University of California, Berkeley \\ RISHI SAKET, Princeton University \\ YI WU, IBM Almaden Research Center
}

The Unique Games Conjecture (UGC) has emerged in recent years as the starting point for several optimal inapproximability results. While for none of these results a reverse reduction to Unique Games is known, the assumption of bijective projections in the Label Cover instance nevertheless seems critical in these proofs. In this work, we bypass the need for UGC assumption in inapproximability results for two geometric problems, obtaining a tight NP-hardness result in each case.

The first problem, known as $L_{p}$ Subspace Approximation, is a generalization of the classic least squares regression problem. Here, the input consists of a set of points $X=\left\{a_{1}, \ldots, a_{m}\right\} \subseteq \mathbb{R}^{n}$ and a parameter $k$ (possibly depending on $n$ ). The goal is to find a subspace $H$ of $\mathbb{R}^{n}$ of dimension $k$ that minimizes the $\ell_{p}$ norm of the Euclidean distances to the points in $X$. For $p=2, k=n-1$, this reduces to the least squares regression problem, while for $p=\infty, k=0$ it reduces to the problem of finding a ball of minimum radius enclosing all the points. We show that for any fixed $p \in(2, \infty)$, and for $k=n-1$, it is NP-hard to approximate this problem to within a factor of $\gamma_{p}-\epsilon$ for constant $\epsilon>0$, where $\gamma_{p}$ is the $p$ th norm of a standard Gaussian random variable. This matches the $\gamma_{p}$ approximation algorithm obtained by Deshpande, Tulsiani, and Vishnoi who also showed the same hardness result under the UGC.

The second problem we study is the related $L_{p}$ Quadratic Grothendieck Maximization Problem, considered by Kindler, Naor, and Schechtman. Here, the input is a multilinear quadratic form $\sum_{i, j=1}^{n} a_{i j} x_{i} x_{j}$ and the goal is to maximize the quadratic form over the $\ell_{p}$ unit ball, namely, all $x$ with $\sum_{i=1}^{n}\left|x_{i}\right|^{p} \leqslant 1$. The problem is polynomial time solvable for $p=2$. We show that for any constant $p \in(2, \infty)$, it is NP-hard to approximate the quadratic form to within a factor of $\gamma_{p}^{2}-\epsilon$ for any $\epsilon>0$. The same hardness factor was shown under the UGC by Kindler et al. We also obtain a $\gamma_{p}^{2}$-approximation algorithm for the problem using the convex relaxation of the problem defined by Kindler et al. A $\gamma_{p}^{2}$ approximation algorithm has also been independently obtained by Naor and Schechtman.

These are the first approximation thresholds, proven under $\mathrm{P} \neq \mathrm{NP}$, that involve the Gaussian random variable in a fundamental way. Note that the problem statements themselves do not explicitly involve the Gaussian distribution.

CCS Concepts: $\bullet$ Theory of computation $\rightarrow$ Problems, reductions and completeness; Rounding techniques; Numeric approximation algorithms; • Mathematics of computing $\rightarrow$ Computations on matrices; Convex optimization; Quadratic programming; Interpolation;

Additional Key Words and Phrases: NP-hardness, Grothendieck, subspace approximation, label cover

V. Guruswami was supported in part by a Packard Fellowship, P. Raghavendra was supported by an NSF Career Award and the Alfred. P. Sloan Fellowship, and R. Saket was supported in part by NSF 0832797.

Authors' addresses: V. Guruswami, Computer Science Department, Gates-Hillman Complex, 7211 Carnegie Mellon University, 5000 Forbes Avenue, Pittsburgh PA 15213; email: guruswami@cs.cmu.edu; P. Raghavendra, Computer Science Division, University of California, Berkeley; email: prasad@cs.berkeley.edu; R. Saket, Researcher, India Research Laboratory, Bangalore, India; email: rissaket@in.ibm.com; Y. Wu, Google Mountain View, 1600 Amphitheatre Parkway, Mountain View, CA 94043; email: wuyish@gmail.com.

Permission to make digital or hard copies of part or all of this work for personal or classroom use is granted without fee provided that copies are not made or distributed for profit or commercial advantage and that copies show this notice on the first page or initial screen of a display along with the full citation. Copyrights for components of this work owned by others than ACM must be honored. Abstracting with credit is permitted. To copy otherwise, to republish, to post on servers, to redistribute to lists, or to use any component of this work in other works requires prior specific permission and/or a fee. Permissions may be requested from Publications Dept., ACM, Inc., 2 Penn Plaza, Suite 701, New York, NY 10121-0701 USA, fax +1 (212) 869-0481, or permissions@acm.org.

(c) 2016 ACM 1549-6325/2016/02-ART6 $\$ 15.00$

DOI: http://dx.doi.org/10.1145/2737729 
ACM Reference Format:

Venkatesan Guruswami, Prasad Raghavendra, Rishi Saket, and Yi Wu. 2016. Bypassing UGC from some optimal geometric inapproximability results. ACM Trans. Algorithms 12, 1, Article 6 (February 2016), 25 pages.

DOI: http://dx.doi.org/10.1145/2737729

\section{INTRODUCTION}

The Unique Games Conjecture of Khot [2002b] asserts that a certain binary constraint satisfaction problem is hard to approximate over a large enough alphabet. The conjecture has been shown in recent years to imply optimal hardness results for various important combinatorial optimization problems such as Maximum Cut [Khot et al. 2007], Vertex Cover [Khot and Regev 2008], and more generally, constraint satisfaction problems [Raghavendra 2008] and ordering problems [Guruswami et al. 2011]. However, arguably there has been little progress toward proving the conjecture. On the contrary, recent algorithmic results have disproved some stronger variants of the conjecture [Arora et al. 2010], and solved the Unique Games problem on special classes of instances like expanders [Arora et al. 2008], and more generally, graphs with few "bad" eigenvalues [Guruswami and Sinop 2011; Barak et al. 2011]. Moreover, while the Unique Games Conjecture is known to imply optimal inapproximability results for Maximum Cut, Vertex Cover, and several other problems, the converse is unknown in each case. (In other words, we only know Unique-Games hardness results, but no "Unique-Games completeness" results.) This leaves the possibility open that while the implications of the conjecture are true, the conjecture itself is false.

For all these reasons, it is a worthwhile endeavor to investigate if the optimal inapproximability results obtained via the Unique Games Conjecture can be shown without appealing to the conjecture. In this work, we consider two geometric problems for which optimal inapproximability results based on the Unique Games Conjecture have been shown previously, and obtain the same NP-hardness results unconditionally, that is, without appealing to the conjecture.

\subsection{Our Main Results}

$L_{p}$ Subspace Approximation Problem. The first problem we consider is the $L_{p}$ Subspace Approximation Problem for $2<p<\infty$-a natural generalization of the least squares regression problem, the low rank matrix approximation problem, and the problem of computing radii of point sets. Here, the input consists of a set of points $X=\left\{a_{1}, \ldots, a_{m}\right\} \subseteq \mathbb{R}^{n}$, and an integer $1 \leqslant k \leqslant n$. The goal is to find a $k$-dimensional subspace $H$ of $\mathbb{R}^{n}$ that minimizes the $\ell_{p}$ norm of the Euclidean distances to the points in $X$. Formally, the goal is to compute

$$
\operatorname{Sub}_{p}(X, k):=\min _{\substack{H \subset \mathbb{R}^{n}: \\ \operatorname{dim}(H)=k}}\left(\sum_{i=1}^{m} \operatorname{dist}\left(H, a_{i}\right)^{p}\right)^{1 / p},
$$

where dist(, ) is the usual $\ell_{2}$ distance between a subspace and a point. Informally, it is the problem of determining how close a given set of points is from lying in a smaller subspace, where the measure of closeness to a subspace is the $\ell_{p}$ norm of the tuple of Euclidean distances of the set of points from the subspace. Such problems arise naturally in classification of large data-sets for applications in machine learning and data mining. As an algorithmic question, it is a generalization of various special cases for different values of $p$ such as low rank matrix approximation $(p=2)$ or computing the radii of point sets $(p=\infty)$. We refer the reader to Deshpande et al. [2011] for a more comprehensive discussion of these connections. 
In this work, we focus on the hardness of approximating the $L_{p}$ Subspace Approximation Problem for the case when $k=n-1$, that is, the problem of finding a hyperplane that is closest to the set of points in the measure defined previously. Let the $p$ th norm of a normal random variable be denoted by $\gamma_{p}$. Formally, for any $p \geqslant 0, \gamma_{p}:=\left(\mathbb{E}\left[|g|^{p}\right]\right)^{1 / p}$, where $g$ is a Gaussian random variable with mean 0 and variance 1. Asymptotically, as $p \rightarrow \infty, \gamma_{p}=\sqrt{\frac{p}{e}}(1+o(1))$. Recently, Deshpande et al. [2011] obtained a $\gamma_{p}$ approximation for the problem, and showed a matching hardness assuming the Unique Games Conjecture (UGC). Bypassing the need for the UGC, we obtain a $(1-\epsilon) \gamma_{p}$ NP-hardness of approximation unconditionally. The following theorem is proved in Section 4.

Theorem 1.1. For any constants $p \in(2, \infty)$ and $\epsilon>0$, the $L_{p}$ Subspace Approximation Problem for $k=n-1$ is NP-hard to approximate within a factor of $(1-\epsilon) \gamma_{p}$.

$L_{p}$ Grothendieck Problem. The second problem we consider is that of maximizing a multilinear quadratic form over the unit $\ell_{p}$ ball in $\mathbb{R}^{n}$ for constant $p, 2<p<\infty$. Formally, the input to the problem is a symmetric $n \times n$ matrix $A=\left(a_{i j}\right)$ with zero diagonal entries, and the goal is to compute the following quantity:

$$
\operatorname{Val}_{p}(A):=\max \left\{\left.\sum_{i, j=1}^{n} a_{i j} x_{i} x_{j}\left|\sum_{i}\right| x_{i}\right|^{p} \leqslant 1\right\} .
$$

We refer to this problem as the $L_{p}$ Quadratic Grothendieck Maximization Problem. In the case where $p=2, \operatorname{Val}_{2}(A)$ is nothing but the maximum eigenvalue of the matrix $A$ and hence is computationally tractable. The case $p=\infty$ is commonly referred to as the quadratic Grothendieck problem and has been extensively studied (especially the bipartite case, that is, when $A$ is the adjacency matrix of a weighted bipartite graph) in mathematics and computer science for its applications to combinatorial optimization, graph theory, and correlation clustering [Nemirovski et al. 1999; Alon and Naor 2006; Alon et al. 2006; Charikar and Wirth 2004]. The case when $2<p<\infty$ has applications toward studying spin glass systems in physics (see Kindler et al. [2010]).

Kindler et al. [2010] obtained a $\left(\gamma_{p}^{2}-\epsilon\right)$-hardness for every $\epsilon>0$ for the $L_{p}$ Quadratic Grothendieck problem assuming the UGC, and also exhibited an almost matching $\frac{p}{e}+$ $30 \log p$-approximation algorithm. Bypassing the Unique Games Conjecture, we obtain a $\gamma_{p}^{2}$ NP-hardness of approximation unconditionally for the problem. We also obtain an approximation algorithm that exactly matches the hardness result for every $p$.

Theorem 1.2. For any constants $p \in(2, \infty)$ and $\epsilon>0$, it is NP-hard to approximate $\operatorname{Val}_{p}(A)$ for a symmetric matrix A with all diagonal entries zero to within a factor of $\left(\gamma_{p}^{2}-\epsilon\right)$.

THEOREm 1.3. There is a polynomial time algorithm to approximate $\operatorname{Val}_{p}(A)$ to within $\gamma_{p}^{2}$ for any symmetric matrix A with all diagonal entries equal to zero.

A $\gamma_{p}^{2}$ approximation for $\operatorname{Val}_{p}(A)$ has also been independently obtained by Naor and Schechtman [2010] as part of a more general result.

Remark. Following convention from previous work [Kindler et al. 2010], we require that the input matrix $A$ to the $L_{p}$ Quadratic Grothendieck Maximization Problem to have zero on the diagonal entries. The origins of this convention can be traced back to the well studied case of $p=\infty$. In this case, if $A$ has nonnegative diagonal entries, the problem reduces to maximizing the quadratic form over $\{-1,1\}$-valued variables. The diagonal terms will contribute a fixed value to the objective for every $\{-1,1\}$ solution and thus it is natural to study the approximation ratio on instances with zero 
diagonals. In Section 5.2, we will present a reduction from arbitrary instances with nonzero diagonal entries, to instances with zeros on the diagonal.

\subsection{Discussion of Our Results}

The previously mentioned NP-hardness results are noteworthy for the following reasons.

First, the inapproximability factors for both problems are irrational numbers arising from the Gaussian distribution, although neither of the problems involve the Gaussian distribution directly. Inapproximability factors arising from properties of the Gaussian distribution have previously been obtained for other problems-such as Maximum Cut [Khot et al. 2007] —using the Unique Games Conjecture. These reductions based on the UGC naturally involve the Gaussian distribution via analytic tools such as the Invariance Principle [Mossel 2010].

Second, the inapproximability factors obtained in each case arise directly from a semidefinite program for the problem. Again, the optimality of semidefinite programs has been a recurring theme in the UGC based hardness results, while this work is among the few NP-hardness results that highlight this phenomenon. In addition, almost all these few known tight NP-hardness of approximation results have ratios being "nice" numbers, such as $2 / 3$ for the Majority function on three variables and $1 / 2$ for Max 3CSPs [Zwick 1998]. In comparison, our thresholds are irrational and involve the Gaussian distribution in a fundamental way. These properties of the thresholds suggest that it is unlikely an alternative simple combinatorial algorithm will achieve the same approximation ratio.

Third, the reductions in this work are based on a dictatorship test that quantitatively utilizes the Central Limit Theorem, which states that the distribution of a sum of a significant number of independent Bernoulli random variables is close to a Gaussian distribution. This is precisely the reason for the appearance of the Gaussian distribution. A key ingredient in our reductions is the smooth version of Label Cover that enables us to devise a more sophisticated decoding procedure that can be combined with the dictatorship test. It is pertinent to note that a couple of the (few) previous results using smooth versions of Label Cover, on hardness of learning intersection of halfspaces [Khot and Saket 2011] and monomials [Feldman et al. 2009], have also used analysis based on versions of the Central Limit Theorem. Our results imply that for many geometric (and possibly other combinatorial optimization) problems, using Unique Games Conjecture is not necessary and the Smooth Label Cover (which is NPhard) suffices in its place. Technically speaking, compared with previous work such as Feldman et al. [2009] (which use smoothness of the Label Cover to bound the fourth moments in the invariance analysis), we believe the decoding technique based on smooth Label Cover in this work is conceptually simpler and may be useful for bypassing the UGC from other problems.

Organization of the article. The next section motivates the problems studied in this article and gives an overview of the related previous work and informal description of the techniques used to prove our results. Section 3 defines the problems formally and reviews the necessary technical tools. Theorems 1.1 and 1.2 are proved in Sections 4 and 5, respectively. Section 6 gives the approximation algorithm proving Theorem 1.3.

\section{MOTIVATION AND RELATED WORK}

$L_{p}$ Subspace Approximation Problem. Algorithmically, various special cases of this problem have been well studied. For $p=2$ it reduces to the problem of determining a rank $k$ approximation $B$ to an $n \times m$ matrix $A$ with respect to the Frobenius norm, which can be computed in polynomial time by using the Singular Value Decomposition of $A$ [Golub and van Loan 1996]. Efficient $(1+\epsilon)$ approximations have been given various 
cases such as, for $p=1$ and constant $k$ [Feldman et al. 2010]; $p=\infty$ and constant $k$ [Har-Peled and Varadarajan 2002]; and for general $p$ and constant $k$ [Shyamalkumar and Varadarajan 2007; Deshpande and Varadarajan 2007]. On the other hand, the problem can be approximated to within $O(\sqrt{\log m})$ for any value of $k$ for $p=\infty$ as shown in Varadarajan et al. [2007] building on Nemirovski et al. [1999].

On the complexity front, Brieden et al. [2000] showed that the problem is NP-hard to solve optimally for $k=n-1$ and $p=\infty$. Subsequently, the problem was shown to be NP-hard to approximate within $(\log m)^{\delta}$, where $\delta>0$ is a fixed constant, and $k \leqslant n-n^{\epsilon}$ for any $0<\epsilon<1$ and $p=\infty$ [Varadarajan et al. 2007].

In more recent work, Deshpande et al. [2011] gave a $\sqrt{2} \gamma_{p}$ approximation for this problem for any $k$ and any $p \geqslant 2$, and a $\gamma_{p}$ approximation factor when $k=n-1$. Assuming the Unique Games Conjecture they also prove that the problem is hard approximate within a factor of $(1-\epsilon) \gamma_{p}$ when $k=n-1$.

$L_{p}$ Quadratic Grothendieck Maximization Problem. The special case of the problem when $p=\infty$ (maximizing over the hypercube), has been extensively studied. The problem is known to admit an $O(\log n)$ approximation [Nesetrov 1998; Nemirovski et al. 1999; Megretski 2001; Charikar and Wirth 2004]. On the other hand, it was shown to be NP-hard to approximate within some constant factor [Alon and Naor 2006; Charikar and Wirth 2004]. Subsequent work [Arora et al. 2005] gave the best known inapproximability factor of $(\log n)^{c}$ for some $c>0$ for this problem.

The $L_{p}$ Quadratic Grothendieck Maximization Problem for constant $p$ such that $2<p<\infty$ has received attention more recently in the work of Kindler et al. [2010]. They exhibit an algorithm to approximate $\operatorname{Val}_{p}(A)$ to within a factor of $\frac{p}{e}+30 \log p$ and also show a Unique Games Conjecture [Khot 2002b] based inapproximability factor of $\gamma_{p}^{2}-\epsilon$ for all $\epsilon>0$. Here, $\gamma_{p}$ denotes the $p$ th norm of a standard Gaussian variable. Note that while asymptotically (i.e., as $p \rightarrow \infty$ ) the upper and lower bounds both tend to $\frac{p}{e}(1+o(1))$, for a fixed constant $p(2<p<\infty)$, there remained a gap between them.

\subsection{Overview of the Techniques}

In the next few paragraphs we give an informal description of the techniques used in proving the results of the article and the new ingredients employed to build upon previous work [Kindler et al. 2010; Deshpande et al. 2011].

NP-Hardness Reductions. Both hardness of approximation results shown in this work are obtained via reductions from the Smooth Label Cover problem, which is a variant of the well-known Label Cover problem. The Label Cover problem, a bipartite constraint satisfaction problem (CSP) with constraints on pairs of vertices, is the starting point of a majority of reductions showing hardness of approximation. A Label Cover instance consists of a bipartite graph with vertex sets $U V$, with label sets $[N]=\{1, \ldots, N\}$ and $[M]=\{1, \ldots, M\}$ for $U$ and $V$, respectively, where $M \geqslant N$. Every edge $e=(u, v)$ $\left(u \in U\right.$ and $v \in V$ ) has a corresponding projection $\pi^{e}:[M] \rightarrow[N]$, and is satisfied by a labeling consistent with $\pi^{e}$. The goal is to find a labeling satisfying the maximum number of edges. Very strong hardness of approximation results are known for the Label Cover problem, and these in turn are the starting point of almost all hardness of approximation results.

Unique Games is a special case of Label Cover where $M=N$ and every edge has a bijective constraint, that is, the label of either end point determines the unique label to the other that satisfies the edge. While bijective constraints are convenient for showing hardness results, the NP-hardness of approximating Unique Games is still conjectural.

Smooth Label Cover is a variant of Label Cover which, while having properties similar to Unique Games, can still be shown to be NP-hard to approximate. It was first introduced in Khot [2002a] for proving hardness results in hypergraph coloring and 
subsequently utilized for other applications [Khot and Saket 2006, 2011; Feldman et al. 2009]. Roughly speaking, in a Smooth Label Cover instance, for every vertex $v \in V$, the set of projections corresponding to the edges incident on $v$ forms a good hash family. More precisely, fix a vertex $v \in V$ and let $a$ and $b$ be any two of its labels. Then, with high probability over the choice of a random edge $e$ incident on $v$, the projection $\pi^{e}$ maps $a$ and $b$ to distinct labels. This is weaker than Unique Games where all projections are bijections and therefore the stated property holds with probability 1 over the choice of $e$.

$L_{p}$ Subspace Approximation Problem. Now we describe the reduction from Smooth Label Cover to $L_{p}$ Subspace Approximation.

Analogously to the construction of Deshpande et al. [2011], we formulate the Label Cover instance as a system of linear equations. More precisely, there is a coordinate for every label of every vertex of the Smooth Label Cover instance. The constraints between the labels of vertices are translated to homogeneous linear equations between the corresponding variables. Let $F$ denote the vector space of solutions to this linear system. The $L_{p}$ Subspace Approximation instance we construct will lie entirely in this subspace $F$. This method of enforcing constraints of Label Cover is known as folding [Gopalan et al. 2010]. Not every vector in $F$ corresponds to a labeling of the Smooth Label Cover instance. In particular, every vertex can be assigned at most one label-the vector corresponding to a labeling is necessarily sparse. Hence, the goal of solving Smooth Label Cover reduces to the problem of finding a sparse vector in the linear space $F$.

The instance will consist of a set of points $X$ inside $F$, such that if $\langle\mathbf{b}, \mathbf{x}\rangle=0$ is a hyperplane close in the appropriate norm ( $\ell_{p}$ norm of the Euclidean distances $)$ to $X$, then $\mathbf{b}$ is a sparse vector in $F$. To this end, for every vertex $v$ in the Smooth Label Cover, we include $2^{M}$ points in $F$ as follows: for each setting of the $[M]$ coordinates corresponding to $v$ from $\{-1,1\}$ with the coordinates for all the other vertices set to 0 , project the resulting vector into $F$. This construction follows closely the construction in Deshpande et al. [2011], except for the folding step where the vector is projected into the linear space $F$. Folding could not be used in Deshpande et al. [2011] since Unique Games does not have perfect completeness (optimal solution need not satisfy all edges) and hence cannot be translated into a system of linear equations.

The crucial property that is used in the analysis is the Berry-Esséen theorem. For a real vector $\mathbf{b} \in \mathbb{R}^{M},\langle\mathbf{b}, \mathbf{x}\rangle$ over a random $\mathbf{x} \in\{-1,1\}^{M}$ is distributed as a $\{-1,1\}$ random variable if $\mathbf{b}$ corresponds to a valid assignment. Hence, if $\mathbf{b}$ corresponds to a valid assignment, then the $L_{p}$ norm of $\langle\mathbf{b}, \mathbf{x}\rangle$ is at most 1 . On the other hand, by the Berry-Esséen theorem, if $\mathbf{b}$ is not sparse, then $\langle\mathbf{b}, \mathbf{x}\rangle$ is distributed like a Gaussian, thereby having an $L_{p}$ norm of $\gamma_{p}$. This lies at the core of the $\gamma_{p}$-hardness result for $L_{p}$ subspace approximation. The difficulty in the analysis is to show the converse that, every vector $\mathbf{b}$ that is somewhat sparse ( $\langle\mathbf{b}, \mathbf{x}\rangle$ not distributed as a Gaussian) corresponds to a good labeling of the Label Cover instance. Smoothness of the Label Cover instance plays a crucial role in showing this fact.

$L_{p}$ Quadratic Grothendieck Maximization Problem. For the $L_{p}$ Quadratic Grothendieck Problem our overall approach is inspired by Kindler et al. [2010], but carried out in a modular and general setting. Specifically, we exhibit a black-box reduction from the $L_{p}$ subspace approximation problem to a variant of the $L_{p}$ Quadratic Grothendieck problem. In this variant, the desired solution is constrained to be within a subspace that is part of the input.

We proceed to reduce this problem to the standard variant of the $L_{p}$ Quadratic Grothendieck problem in two steps. In the first step, we will show how to eliminate the constraint that the solution lies in a subspace. We achieve this by including a large 
penalty term into the objective, which precludes the optimal solution from being far from the subspace. In the second step, we eliminate the nonzero entries on the diagonal. To this end, we will replace each variable by a big block of variables, so that the relative contribution of the diagonal terms becomes negligible.

Approximating the $L_{p}$ Quadratic Grothendieck Maximization Problem. Our algorithm is essentially a simplification of the techniques in Kindler et al. [2010]. We define the following relaxation for $\operatorname{Val}_{p}(A)$ :

$$
\operatorname{Vec}_{p}(A):=\max \left\{\sum_{i, j=1}^{n} a_{i j}\left\langle v_{i}, v_{j}\right\rangle \mid\left\{v_{1}, \ldots, v_{n}\right\} \subseteq L_{2}, \sum_{i=1}^{n}\left\|v_{i}\right\|_{2}^{p} \leqslant 1\right\} .
$$

As observed in Kindler et al. [2010], the preceding convex program can be solved in polynomial time to arbitrary precision. We directly $\operatorname{show}_{\text {that }} \operatorname{Vec}_{p}(A)$ is a $\gamma_{p}^{2}$ approximation to $\operatorname{Val}_{p}(A)$. This can be easily derived from the following fact: there exist mean zero Gaussian random variables $h_{i}$ for $i=1, \ldots, n$ such that $\mathbb{E}\left[h_{i} h_{j}\right]=\left\langle v_{i}, v_{j}\right\rangle$. Writing $\operatorname{Vec}_{p}(A)$ as $\mathbb{E}\left[\sum_{i, j=1}^{n} a_{i j} h_{i} h_{j}\right]$ and normalizing each variable by $\left(\sum_{k=1}^{n}\left|h_{k}\right|^{p}\right)^{1 / p}$ yields the desired approximation. This differs from the proof in Kindler et al. [2010], which obtains a slightly weaker approximation via a truncation based rounding algorithm. In an independent work, Naor and Schechtman [2010] obtain an approximation for a more general version of the problem associated with arbitrary convex bodies. For the special case of the unit $\ell_{p}(p>2)$ balls, this reduces to an approximation identical to the one we present. Our proof also yields a polynomial time rounding algorithm to compute a solution $\left\{x_{i}\right\}_{i=1}^{n}$, which approximates $\operatorname{Val}_{p}(A)$ to within a factor of $\gamma_{p}^{2}(1+\delta)$ for arbitrarily small $\delta>0$.

\section{PRELIMINARIES}

We begin this section by first formally defining the two problems that we study.

Definition 3.1. The $L_{p}$ Subspace Approximation Problem, which we denote by $\operatorname{Subspace}(k, p)$ where $k$ is a parameter (possibly depending on $n$ ) is as follows: given a set of points $X=\left\{a_{1}, \ldots, a_{m}\right\} \subseteq \mathbb{R}^{n}$, to compute the following quantity

$$
\operatorname{Sub}_{p}(X, k):=\min _{\substack{H \subseteq \mathbb{R}^{n}: \\ \operatorname{dim}(H)=k}}\left(\sum_{i=1}^{m} \operatorname{dist}\left(H, a_{i}\right)^{p}\right)^{1 / p},
$$

where the minimum is taken over all $k$-dimensional subspaces of $\mathbb{R}^{n}$ and $\operatorname{dist}(H, a)$ is the minimum Euclidean distance between $a$ and any point in $H$.

Definition 3.2. The $L_{p}$ Quadratic Grothendieck Maximization Problem, which we denote as $\mathrm{QM}(p)$ for $1 \leqslant p<\infty$, is as follows: given a symmetric matrix $A \in \mathbb{R}^{n \times n}$ with diagonal entries all zero, to compute the following quantity,

$$
\operatorname{Val}_{p}(A):=\max \left\{\left.\sum_{i, j=1}^{n} a_{i j} x_{i} x_{j}\left|\sum_{i=1}^{n}\right| x_{i}\right|^{p} \leqslant 1\right\} .
$$

We denote by $\gamma_{p}$ the $p$ th norm of a standard Gaussian random variable, that is, for any $p \geqslant 0, \gamma_{p}:=\left(\mathbb{E}\left[|g|^{p}\right]\right)^{1 / p}$, where $g$ is a Gaussian random variable with mean 0 and variance 1 . The analysis of the dictatorship tests in our reductions requires lower bounds on the norms of sums of independent variables. The following lemma, proved in Kindler et al. [2010] (as Lemma 2.5) gives us the required bound. 
Lemma 3.3. Let $X_{1}, \ldots, X_{n}$ be independent random variables such that $\mathbb{E}\left[X_{i}\right]=0$ for all $1 \leqslant i \leqslant n$ and $\sum_{j=1}^{n} \mathbb{E}\left[X_{j}^{2}\right]=1$. Assume that for some $\tau \in\left(0, e^{-4}\right)$, we have $\sum_{j=1}^{n} \mathbb{E}\left[\left|X_{j}\right|^{3}\right] \leqslant \tau$. Then for every $p \geqslant 1$,

$$
\left(\mathbb{E}\left[\left|\sum_{j=1}^{n} X_{j}\right|^{p}\right]\right)^{1 / p} \geqslant \gamma_{p} \cdot\left(1-4 \tau(\log (1 / \tau))^{p / 2}\right) .
$$

\subsection{Smooth Label Cover}

Our reductions require a special (not necessarily bipartite) variant of the usual Label Cover problem, which is formally defined as follows.

Definition 3.4. An instance of Smooth Label Cover $\mathcal{L}\left(G(V, E), N, M,\left\{\pi^{e, v} \mid e \in E\right.\right.$, $v \in e\}$ ) consists of a regular connected (undirected) graph $G(V, E)$ with vertex set $V$ and edge set $E$. Every edge $e=\left(v_{1}, v_{2}\right)$ is associated with functions $\left\{\pi^{e, v_{i}}\right\}_{i=1}^{2}$ where $\pi^{e, v_{i}}:[M] \rightarrow[N]$. A vertex labeling is a mapping defined on $L: V \rightarrow[M]$. A labeling $L$ satisfies edge $e=\left(v_{1}, v_{2}\right)$ if $\pi^{e, v_{1}}\left(L\left(v_{1}\right)\right)=\pi^{e, v_{2}}\left(L\left(v_{2}\right)\right)$. The goal is to find a labeling that satisfies the maximum number of edges.

The following theorem states the hardness of approximation for the Smooth Label Cover problem and also describes the various structural properties, including smoothness, that are satisfied by the hard instances. A proof of the theorem is included in Appendix A.

THEOREM 3.5. There exists a constant $c_{0}>0$ such that for any constant integer parameters $J, R \geqslant 1$, it is NP-hard to distinguish between the following two cases for a Smooth Label Cover instance $\mathcal{L}\left(G(V, E), N, M,\left\{\pi^{e, v} \mid e \in E, v \in e\right\}\right)$ with $M=7^{(J+1) R}$ and $N=2^{R} 7^{J R}$ :

-(YES Case) There is a labeling that satisfies every edge.

-(NO Case) Every labeling satisfies less than a fraction $2^{-c_{0} R}$ of the edges.

In addition, the instance $\mathcal{L}$ satisfies the following properties:

-(Smoothness) For any vertex $w \in V, \forall i, j \in[M], i \neq j, \operatorname{Pr}_{e \sim w}\left[\pi^{e, w}(i)=\pi^{e, w}(j)\right] \leqslant$ $1 / J$, where the probability is over a randomly chosen edge incident on $w$.

-The degree of the (regular) graph $G$, which we denote by $d$, is a constant depending only on $R$ and $J$.

-For any vertex $v$, edge e incident on $v$, and any element $i \in[N]$, we have $\left|\left(\pi^{e, v}\right)^{-1}(i)\right| \leqslant$ $t:=4^{R}$; that is, there are at most $t=4^{R}$ elements in $[M]$ that are mapped to the same element in $[N]$.

-(Weak Expansion) For any $\delta>0$, let $V^{\prime} \subseteq V$ and $\left|V^{\prime}\right|=\delta \cdot|V|$, then the number of edges among the vertices in $\left|V^{\prime}\right|$ is at least $\delta^{2}|E|$.

The hard instances of Smooth Label Cover obtained in Theorem 3.5 also contain a negligible fraction of self-loops. These are satisfied by any labeling, and are ignored in the hardness reductions.

Note on notation. In the following sections, the parameter $n$ need not denote the size of the instances and its definition will be made clear at the beginning of each section.

\section{HARDNESS REDUCTION FOR $L_{P}$ SUBSPACE APPROXIMATION}

In this section, we describe the NP-hardness reduction from Smooth Label Cover to the $L_{p}$ Subspace Approximation Problem for a fixed $p>2$. Specifically, we will show the following. 
Theorem 4.1. For any fixed $p>2$ and $\epsilon>0$, there is a polynomial time reduction from an instance $\mathcal{L}$ of Smooth Label Cover with appropriately chosen parameters $J$ and $R$ to a set of points $X \subseteq \mathbb{R}^{\mathcal{N}}$ as an instance of $\operatorname{Subspace}(\mathcal{N}-1, p)$ such that

-(Completeness) If $\mathcal{L}$ is a YES instance, then $\operatorname{Sub}_{p}(\mathcal{N}-1, X)=1$.

-(Soundness) If $\mathcal{L}$ is a NO instance, then $\operatorname{Sub}_{p}\left(\mathcal{N}^{p}-1, X\right) \geqslant \gamma_{p}(1-\epsilon)$.

The preceding implies that it is NP-hard to approximate $\operatorname{Subspace}(\mathcal{N}-1, p)$ within a factor of $(1-\epsilon) \gamma_{p}$ for all $\epsilon>0$.

Let the Smooth Label Cover instance be $\mathcal{L}\left(G(V, E), N, M,\left\{\pi^{e, v} \mid e \in E, v \in e\right\}\right)$. We choose the parameters $J$ and $R$ as part of the analysis in Section 4.4. For convenience, let $n:=|V|$. The notation dim is used to denote the dimension of the point set constructed in the reduction and is not explicitly calculated. The set of points constructed is an instance of Subspace $(\operatorname{dim}-1, p)$. The Euclidean distance of a point from a dimension (dim - 1) subspace, that is, a hyperplane through the origin, is the same as the magnitude of the dot product of (the vector defining) that point with the unit normal vector to the subspace. Therefore, the problem Subspace(dim $-1, p$ ) is the same as computing a unit vector that minimizes the sum of the $p$ th powers of the dot products of the given points with the vector. Our reduction will follow this latter formulation, with the goal being to compute such a unit normal vector. The reduction proceeds in two steps: the first step yields a preliminary instance consisting of a set of points and the second step applies a folding operation to generate the final instance.

For notational convenience, in this section we will represent vectors with boldface characters.

\subsection{Step 1: Preliminary Instance $\mathcal{A}_{\text {prel }}$}

We begin by constructing the set of coordinates over which the instance is defined. For any vertex $v \in V$, let $\mathcal{P}_{v}$ be the set of coordinates $\{(v, i) \mid i \in[M]\}$, and $\mathcal{P}=\cup_{v \in V} \mathcal{P}_{v}$. In other words, $\mathcal{P}$ contains a coordinate for every label of every vertex. The instance $\mathcal{A}_{\text {prel }}$ will be over the space $\mathbb{R}^{\mathcal{P}}$ consisting of points constructed as follows.

For every vertex $v \in V$, let the set $X^{v}$ be the set of all points in $\mathbb{R}^{\mathcal{P}}$ that are zero in the coordinates not corresponding to $v$, that is, $\mathcal{P} \backslash \mathcal{P}_{v}$, and take the values $\{-1,1\}$ in the $M$ coordinates $\mathcal{P}_{v}$ corresponding to $v$. More formally,

$X^{v}:=\left\{\mathbf{x} \in \mathbb{R}^{\mathcal{P}} \mid \forall i \in[M], v^{\prime} \in V, \mathbf{x}\left(v^{\prime}, i\right) \in\{-1,1\}\right.$ if $v^{\prime}=v$, and $\mathbf{x}\left(v^{\prime}, i\right)=0$ otherwise $\}$.

The instance $\mathcal{A}_{\text {prel }}$ consists of the point set $X:=\cup_{v \in V} X^{v}$.

Consider a vector $\mathbf{b} \in \mathbb{R}^{\mathcal{P}}$. For any vertex $v \in V$, define $\mathbf{b}_{v}$ to be the vector that is the same as $\mathbf{b}$ in the $M$ coordinates $\mathcal{P}_{v}$ and zero in the rest of the coordinates. It is easy to see that for any $v \in V$,

$$
\mathbb{E}_{\mathbf{x} \in\{-1,1\}^{\mathcal{P}_{v}}}\left[\left\langle\mathbf{b}_{v}, \mathbf{x}\right\rangle^{2}\right]=\left\|\mathbf{b}_{v}\right\|_{2}^{2}=\sum_{j=1}^{M} \mathbf{b}(v, j)^{2} .
$$

Using the preceding definition, given $\mathcal{A}_{\text {prel }}$ as an instance, the problem of Subspace $(\operatorname{dim}-1, k)$ is equivalent to computing a unit normal vector $\mathbf{b}$ that minimizes $\mathbb{E}_{v \in V}\left[\mathbb{E}_{\mathbf{x} \in\{-1,1\}^{p_{v}}}\left[\left|\left\langle\mathbf{b}_{v}, \mathbf{x}\right\rangle\right|^{p}\right]\right]$. More formally, $\mathcal{A}_{\text {prel }}$ as an instance of Subspace $(\operatorname{dim}-1, k)$ is equivalent to the following optimization problem:

$$
\begin{array}{cc} 
& \min \left(\mathbb{E}_{v \in V}\left[\mathbb{E}_{\mathbf{x} \in\{-1,1\}^{\mathcal{P}_{v}}}\left[\left|\left\langle\mathbf{b}_{v}, \mathbf{x}\right\rangle\right|^{p}\right]\right]\right)^{1 / p} \\
\text { subject to } & \mathbb{E}_{v \in V}\left[\left\|\mathbf{b}_{v}\right\|_{2}^{2}\right]=1 .
\end{array}
$$


In the next step, we use folding to implicitly induce additional constraints on the structure of the vector $\mathbf{b}$. This incorporates the projection constraints of the edges and enables a good solution $\mathbf{b}$ to be decoded into a good labeling of the Smooth Label Cover instance $\mathcal{L}$.

\subsection{Step 2: Folding and Final Instance $\mathcal{A}_{\text {final }}$}

For any edge $e=(u, v)$ and element $j \in[N]$, define the vector $\mathbf{h}_{j}^{e}$ as follows:

$$
\mathbf{h}_{j}^{e}(w, i)= \begin{cases}1 & \text { if } w=u \text { and } i \in\left(\pi^{e, u}\right)^{-1}(j) \\ -1 & \text { if } w=v \text { and } i \in\left(\pi^{e, v}\right)^{-1}(j) \\ 0 & \text { otherwise. }\end{cases}
$$

The preceding implies that for any vector $\mathbf{b} \in \mathbb{R}^{\mathcal{P}}$,

$$
\forall e=(u, v) \in E, j \in[N], \quad \mathbf{b} \perp \mathbf{h}_{j}^{e} \Leftrightarrow \sum_{i \in\left(\pi^{e, u}\right)^{-1}(j)} \mathbf{b}(u, i)=\sum_{i^{\prime} \in\left(\pi^{e, v}\right)^{-1}(j)} \mathbf{b}\left(v, i^{\prime}\right) .
$$

We now define the subspace $H$ of $\mathbb{R}^{\mathcal{P}}$ as $H:=\operatorname{span}\left(\left\{\mathbf{h}_{j}^{e} \mid e \in E, j \in[N]\right\}\right)$. Let $\mathbb{R}^{\mathcal{P}}=$ $F \oplus H$ where $F \perp H$ is a subspace of $\mathbb{R}^{\mathcal{P}}$. Here $A \oplus B$, for any two orthogonal subspaces $A$ and $B$, denotes their direct sum, which is the subspace spanned by elements of $A \cup B$. The point set $X$ constructed in Step 1 is folded over $H$, that is, each point in $X$ is replaced (with multiplicity) by its orthogonal projection on $F$. Let the resultant set of points be $\bar{X}$, which constitutes the final instance $\mathcal{A}_{\text {final }}$. The point set as well as the expected solution, say $\mathbf{b}$, are written in some orthonormal basis for $F$. Let $\overline{\mathbf{x}} \in \bar{X}$ be the orthogonal projection of a point $x \in X$ onto the subspace $F$, and let $\mathbf{b} \in F$ be the expected solution. Clearly, we have $\langle\mathbf{b}, \overline{\mathbf{x}}\rangle=\langle\mathbf{b}, \mathbf{x}\rangle$. Also, since $\mathbf{b} \perp H$, we have from Equation (7),

$$
\forall e=(u, v) \in E, j \in[N], \sum_{i \in\left(\pi^{e, u}\right)^{-1}(j)} \mathbf{b}(u, i)=\sum_{i^{\prime} \in\left(\pi^{e, v}\right)^{-1}(j)} \mathbf{b}\left(v, i^{\prime}\right) .
$$

We note that the objective value, which can be written as $\mathbb{E}_{\mathbf{x} \in X}\left[|\langle\mathbf{b}, \mathbf{x}\rangle|^{p}\right]$, is unchanged under transformation of orthonormal basis since it is a function of the inner product of vectors. The folding operation only ensures that the constraints given by Equation (8) are satisfied. Therefore, the instance $\mathcal{A}_{\text {final }}$ of $\operatorname{Subspace}(\operatorname{dim}-1, p)$ is equivalent to the following optimization problem over solutions $\mathbf{b} \in \mathbb{R}^{\mathcal{P}}$ :

$$
\min \left(\mathbb{E}_{v \in V}\left[\mathbb{E}_{\mathbf{x} \in\{-1,1\}^{\mathcal{P}_{v}}}\left[\left|\left\langle\mathbf{b}_{v}, \mathbf{x}\right\rangle\right|^{p}\right]\right]\right)^{1 / p}
$$

s.t.

$$
\begin{aligned}
\mathbb{E}_{v \in V}\left[\left\|\mathbf{b}_{v}\right\|_{2}^{2}\right] & =1 & & \text { and } \\
\sum_{i \in\left(\pi^{e, u}\right)^{-1}(j)} \mathbf{b}(u, i) & =\sum_{i^{\prime} \in\left(\pi^{e, v}\right)^{-1}(j)} \mathbf{b}\left(v, i^{\prime}\right), & & \forall e=(u, v) \in E, j \in[N] .
\end{aligned}
$$

Note that the last condition is equivalent to $\mathbf{b} \perp H$.

\subsection{Completeness}

If the instance $\mathcal{L}$ of Smooth Label Cover is a YES instance, then there is a labeling $L$ of the vertices of $\mathcal{L}$ that satisfies all the edges. Using this we construct a solution $\mathbf{b}^{*}$ to the instance $\mathcal{A}_{\text {final }}$ as follows: for any vertex $v \in V$ and element $i \in M, \mathbf{b}^{*}(v, i)$ is 1 if $L(v)=i$ and 0 otherwise. 
Since $L$ satisfies all edges, $\pi^{e, u}(L(u))=\pi^{e, v}(L(v))$ for all edges $e=(u, v)$. Therefore, it is easy to see that $\mathbf{b} \perp H$. Moreover, since there is exactly one nonzero coordinate corresponding to each vertex on which $\mathbf{b}^{*}$ is 1 , we have $\left\|\mathbf{b}_{v}^{*}\right\|_{2}=1$, for all $v \in V$. Hence,

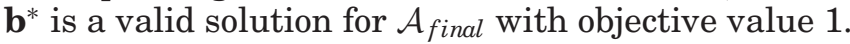

\subsection{Soundness}

We assume, toward a contradiction, that $\mathbf{b} \in \mathbb{R}^{\mathcal{P}}$ is a solution to the instance $\mathcal{A}_{\text {final }}$ such that

$$
\mathbb{E}_{v \in V}\left[\mathbb{E}_{\mathbf{x} \in\{-1,1\}^{p_{v}}}\left[\left|\left\langle\mathbf{b}_{v}, \mathbf{x}\right\rangle\right|^{p}\right]\right] \leqslant \gamma_{p}^{p}(1-\eta),
$$

where $\eta>0$ is a positive constant.

The soundness analysis proceeds by identifying irregular vertices $v$ (which are formally defined later), which have at least one label $i$ such that $|\mathbf{b}(v, i)|$ is significant compared to $\left\|\mathbf{b}_{v}\right\|_{2}$. Vertices $v$ that are not irregular can be shown to satisfy $\mathbb{E}_{\mathbf{x} \in\{-1,1\}^{p_{v}}}\left[\left|\left\langle\mathbf{b}_{v}, \mathbf{x}\right\rangle\right|^{p}\right] \approx \gamma_{p}^{p}\left\|\mathbf{b}_{v}\right\|_{2}^{p}$. This, along with our assumed upper bound on the objective implies that a significant fraction of vertices are irregular. Each irregular vertex $v$ is labeled using an appropriate threshold on the values of $|\mathbf{b}(v, i)|$. The smoothness and the weak expansion properties along with the folding constraints given by Equation (11) ensure that this labeling satisfies a significant fraction of the edges of $\mathcal{L}$, which contradicts the NO case of Theorem 3.5.

The formal definition of an irregular vertex is as follows.

Definition 4.2 ( $\tau$-irregular vertex). A vertex $v \in V$ is said to be $\tau$-irregular if there exists $i \in[M]$ such that $|\mathbf{b}(v, i)|>\tau\left\|\mathbf{b}_{v}\right\|_{2}$. If not, the vertex is referred to as $\tau$-regular.

The following Lemma is an immediate consequence of Lemma 3.3, which is a restatement of Lemma 2.5 in Kindler et al. [2010].

LeMma 4.3. For any constant $p>2$ and every sufficiently small (depending only on p) $\tau>0$ the following holds. If $v \in V$ is $\tau$-regular, then

$$
\mathbb{E}_{\mathbf{x} \in\{-1,1\}^{p_{v}}}\left[\left|\left\langle\mathbf{b}_{v}, \mathbf{x}\right\rangle\right|^{p}\right] \geqslant \gamma_{p}^{p}\left\|\mathbf{b}_{v}\right\|_{2}^{p}(1-\sqrt{\tau}) .
$$

Proof. Let $v$ be $\tau$-regular for a $\tau$ to be chosen later. Let us define the independent random variables $Y_{1}, \ldots, Y_{M}$, where $Y_{i}$ is uniformly distributed in $\left\{-\frac{|\mathbf{b}(v, i)|}{\left\|\mathbf{b}_{v}\right\|_{2}}, \frac{|\mathbf{b}(v, i)|}{\left\|\mathbf{b}_{v}\right\|_{2}}\right\}$ for $i=1, \ldots, M$. Then, $\sum_{i=1}^{M} \mathbb{E}\left[Y_{i}^{2}\right]=1$, and by $\tau$-regularity of $v, \sum_{i=1}^{M} \mathbb{E}\left[\left|Y_{i}\right|^{3}\right] \leqslant \tau$. Note that the left-hand side of the inequality (13) equals $\left\|\mathbf{b}_{v}\right\|_{2}^{p}\left(\mathbb{E}\left[\left|\sum_{i=1}^{M} Y_{i}\right|^{p}\right]\right)$, and the second term in this product can be lower bounded by Lemma 3.3 to obtain

$$
\mathbb{E}_{\mathbf{x} \in\{-1,1\}^{\mathcal{p}_{v}}}\left[\left|\left\langle\mathbf{b}_{v}, \mathbf{x}\right\rangle\right|^{p}\right] \geqslant \gamma_{p}^{p}\left\|\mathbf{b}_{v}\right\|_{2}^{p}\left(1-4 \tau(\log (1 / \tau))^{p / 2}\right)^{p} \geqslant \gamma_{p}^{p}\left\|\mathbf{b}_{v}\right\|_{2}^{p}(1-\sqrt{\tau}),
$$

for a small enough value of $\tau>0$ depending only on $p$.

The next lemma shows that for small enough $\tau$, there is a significant fraction of vertices that are $\tau$-irregular.

LemMa 4.4. For all $p>2$ and $\eta>0$, there are positive constants $\tau$ and $\theta$ depending only on $p$ and $\eta$ such that the following holds. Let $S_{i r r}$ be the set of vertices that are $\tau$-irregular, then $\left|S_{i r r}\right| \geqslant \theta n$.

PRoof. We begin with a lemma upper bounding the squared $\ell_{2}$-norm of blocks of coordinates in $\mathbf{b}$ corresponding to small sets of vertices. This critically depends on the fact that $p>2$.

Lemma 4.5. Let $S \subseteq V$ be a set of size at most $\xi|V|=\xi n$ for some $0 \leqslant \xi \leqslant 1$. Then, $\sum_{v \in S}\left\|\mathbf{b}_{v}\right\|_{2}^{2} \leqslant \gamma_{p}^{2} \xi^{1-2 / \bar{p}_{n}}$. 
Proof. Since the lemma is vacuously true for $\xi=0$, we may assume $\xi>0$. We know from our assumption (Equation (12)) that $\mathbb{E}_{v \in V}\left[\mathbb{E}_{\mathbf{x} \in\{-1,1\}^{\mathcal{P}_{v}}}\left[\left|\left\langle\mathbf{b}_{v}, \mathbf{x}\right\rangle\right|^{p}\right]\right] \leqslant \gamma_{p}^{p}$. This implies

$$
\begin{array}{rlrl}
\gamma_{p}^{p} & \geqslant \mathbb{E}_{v \in V}\left[\left[\left(\mathbb{E}_{\mathbf{x} \in\{-1,1\}^{\mathcal{p}_{v}}}\left[\left|\left\langle\mathbf{b}_{v}, \mathbf{x}\right\rangle\right|^{p}\right]\right)^{1 / p}\right]^{p}\right] & \\
& \geqslant \mathbb{E}_{v \in V}\left[\left[\left(\mathbb{E}_{\mathbf{x} \in\{-1,1\}^{\mathcal{p}_{v}}}\left[\left|\left\langle\mathbf{b}_{v}, \mathbf{x}\right\rangle\right|^{2}\right]\right)^{1 / 2}\right]^{p}\right] & & \text { (using } p>2 \text { and Jensen's inequality) } \\
& =\mathbb{E}_{v \in V}\left[\left\|\mathbf{b}_{v}\right\|_{2}^{p}\right] & & \text { (by Equation (6)) } \\
& \geqslant\left(\frac{|S|}{|V|}\right) \mathbb{E}_{v \in S}\left[\left\|\mathbf{b}_{v}\right\|_{2}^{p}\right] & & \text { (by averaging) } \\
& \geqslant\left(\frac{|S|}{|V|}\right)\left(\mathbb{E}_{v \in S}\left[\left\|\mathbf{b}_{v}\right\|_{2}^{2}\right]\right)^{\frac{p}{2}} & \text { (by Jensen's inequality) } \\
& =\left(\frac{|S|}{|V|}\right)\left[\left(\frac{|V|}{|S|}\right)\left(\frac{\sum_{v \in S}\left\|\mathbf{b}_{v}\right\|_{2}^{2}}{|V|}\right)\right]^{\frac{p}{2}} &
\end{array}
$$

Raising both sides of the final inequality obtained previously to the $(2 / p)$ th exponent and rearranging yields

$$
\sum_{v \in S}\left\|\mathbf{b}_{v}\right\|_{2}^{2} \leqslant \gamma_{p}^{2}\left(\frac{|S|}{|V|}\right)^{1-\frac{2}{p}}|V| \leqslant \gamma_{p}^{2} \xi^{1-\frac{2}{p}} n,
$$

where we use $p>2$ and $|S| \leqslant \xi|V|$. This completes the proof of Lemma 4.5.

Our choice of $\tau>0$ shall be small enough given by Lemma 4.3 to satisfy Equation (13). Let $\theta$ be such that the set of $\tau$-irregular vertices $S_{i r r}$ satisfies $\left|S_{i r r}\right|=\theta n$, then we shall show that $\theta>\theta_{0}$, which is a constant depending only on $\eta$ and $p$. By Lemma 4.5 and Equation (10) we have

$$
\sum_{v \in V \backslash S_{i r r}}\left\|\mathbf{b}_{v}\right\|_{2}^{2} \geqslant n\left(1-\gamma_{p}^{2} \theta^{1-2 / p}\right) .
$$

Also, from our initial assumption on the objective value of $\mathbf{b}$ given by Equation (12) we have

$$
\begin{aligned}
\gamma_{p}^{p}(1-\eta) n & \geqslant \sum_{v \in V} \mathbb{E}_{\mathbf{x} \in\{-1,1\}^{\mathcal{P}_{v}}}\left[\left|\left\langle\mathbf{b}_{v}, \mathbf{x}\right\rangle\right|^{p}\right] \\
& \geqslant \sum_{v \in V \backslash S_{i r r}} \mathbb{E}_{\mathbf{x} \in\{-1,1\}^{p_{v}}}\left[\left|\left\langle\mathbf{b}_{v}, \mathbf{x}\right\rangle\right|^{p}\right] \\
& \geqslant \gamma_{p}^{p}(1-\sqrt{\tau}) n(1-\theta)\left(\frac{1}{(1-\theta) n} \sum_{v \in V \backslash S_{i r r}}\left\|\mathbf{b}_{v}\right\|_{2}^{p}\right) \\
& =\gamma_{p}^{p}(1-\sqrt{\tau}) n(1-\theta) \mathbb{E}_{v \in V \backslash S_{i r r}}\left[\left\|\mathbf{b}_{v}\right\|_{2}^{p}\right] \\
& \geqslant \gamma_{p}^{p}(1-\sqrt{\tau}) n(1-\theta)\left(\mathbb{E}_{v \in V \backslash S_{i r r}}\left\|\mathbf{b}_{v}\right\|_{2}^{2}\right)^{\frac{p}{2}} \\
& \geqslant \gamma_{p}^{p}(1-\sqrt{\tau}) n(1-\theta)\left(1-\gamma_{p}^{2} \theta^{1-2 / p}\right)^{\frac{p}{2}}
\end{aligned}
$$

(by Jensen's inequality)

(by Equation (15)).

From the preceding, choosing $0<\tau \leqslant \eta^{6}$, we obtain that $\theta>\theta_{0}$ for some constant $\theta_{0}$ depending only on $\eta$ and $p$.

We choose appropriately small constants $\tau, \theta>0$ depending only on $p$ and $\eta$ as given by the preceding lemma. To complete the analysis of the soundness, in the rest of this 
section we show that the vector $\mathbf{b}$ can be decoded into a labeling for $\mathcal{L}$ that satisfies a significant fraction of its edges, thereby contradicting the soundness property of Theorem 3.5.

Constructing a good labeling for $\mathcal{L}$. We now describe how to decode the vector $\mathbf{b}$ into a labeling for the set of $\tau$-irregular vertices $S_{i r r}$. Observe that since $\left|S_{i r r}\right| \geqslant \theta n$, by the Weak Expansion property of Theorem 3.5,

$$
\left|E\left(S_{\text {irr }}\right)\right| \geqslant \theta^{2}|E|,
$$

where $E\left(S_{i r r}\right)$ is the set of edges induced by $S_{i r r}$. For every vertex $v \in S_{i r r}$, define

$$
\begin{aligned}
& \Gamma_{0}(v):=\left\{i \in[M]|| \mathbf{b}(v, i) \mid \geqslant \frac{\tau}{2}\left\|\mathbf{b}_{v}\right\|_{2}\right\} \quad \text { and } \\
& \Gamma_{1}(v):=\left\{i \in[M]|| \mathbf{b}(v, i) \mid \geqslant \frac{\tau}{10 t}\left\|\mathbf{b}_{v}\right\|_{2}\right\}
\end{aligned}
$$

where $t=4^{R}$ is the parameter from Theorem 3.5. Clearly, for every vertex $v \in S_{i r r}$ :

$$
\emptyset \neq \Gamma_{0}(v) \subseteq \Gamma_{1}(v), \quad\left|\Gamma_{0}(v)\right| \leqslant \frac{4}{\tau^{2}}, \quad \text { and }\left|\Gamma_{1}(v)\right| \leqslant \frac{100 t^{2}}{\tau^{2}} .
$$

Let $v$ be any vertex in $S_{i r r}$. Call an edge $e$ incident on $v$ to be "good" for $v$ if $\pi^{e, v}$ maps the set $\Gamma_{1}(v)$ injectively (one to one) into $[N]$. Using the smoothness property of Theorem 3.5 along with a union bound over all the pairs of labels in $\Gamma_{1}(v)$ yields the following bound on the probability that a random edge incident on $v$ is "good":

$$
\mathbf{P r}_{e \ni v}[e \text { is "good" for } v] \geqslant 1-\frac{\left|\Gamma_{1}(v)\right|^{2}}{J} \geqslant 1-\frac{10,000 t^{4}}{\tau^{4} J}=: 1-\zeta .
$$

Since the graph of $\mathcal{L}$ is regular, this implies that the total number of edges induced by $S_{i r r}$ that are not "good" for at least one of the end points in $S_{i r r}$ is at most $2 \zeta|E|$. Let $E^{\prime} \subseteq E\left(S_{i r r}\right)$ be the set of edges induced by $S_{i r r}$ that are "good" for both endpoints. The preceding bounds combined with Equation (16) imply that $\left|E^{\prime}\right| \geqslant\left(\theta^{2}-2 \zeta\right)|E|$. The following lemma shows that the folding constraints enforce a structural property on the sets $\Gamma_{0}(v)$ with respect to the edges in $E^{\prime}$.

Lemma 4.6. Let $e=(u, v)$ be any edge in $E^{\prime}$. Then, $\pi^{e, u}\left(\Gamma_{0}(u)\right) \cap \pi^{e, v}\left(\Gamma_{0}(v)\right) \neq \emptyset$.

Proof. Clearly, $u$ and $v$ are $\tau$-irregular. Without loss of generality, assume that $\left\|\mathbf{b}_{u}\right\|_{2} \geqslant\left\|\mathbf{b}_{v}\right\|_{2}$. Since $u$ is $\tau$-irregular, there is a coordinate $\left(u, i_{u}\right)\left(i_{u} \in[M]\right)$ such that $\left|\mathbf{b}\left(u, i_{u}\right)\right| \geqslant \tau\left\|\mathbf{b}_{u}\right\|_{2}$. By construction, $i_{u} \in \Gamma_{0}(u)$.

Let $j_{0}:=\pi^{e, u}\left(i_{u}\right)$. Since $e \in E^{\prime},\left(\pi^{e, u}\right)^{-1}\left(j_{0}\right) \cap \Gamma_{1}(u)=\left\{i_{u}\right\}$. This implies that for all $i \in$ $\left(\pi^{e, u}\right)^{-1}\left(j_{0}\right)$ and $i \neq i_{u},|\mathbf{b}(u, i)|<\frac{\tau}{10 t}\left\|\mathbf{b}_{u}\right\|_{2}$. Moreover, from Theorem $3.5\left|\left(\pi^{e, u}\right)^{-1}\left(j_{0}\right)\right| \leqslant t$. Combining these observations yields

$$
\left|\sum_{i \in\left(\pi^{e, u}\right)^{-1}\left(j_{0}\right)} \mathbf{b}(u, i)\right| \geqslant\left(\tau-t\left(\frac{\tau}{10 t}\right)\right)\left\|\mathbf{b}_{u}\right\|_{2}=\left(\frac{9 \tau}{10}\right)\left\|\mathbf{b}_{u}\right\|_{2} .
$$

We next show that $\left(\pi^{e, v}\right)^{-1}\left(j_{0}\right) \cap \Gamma_{0}(v) \neq \emptyset$, which would imply that $j_{0} \in \pi^{e, u}\left(\Gamma_{0}(u)\right) \cap$ $\pi^{e, v}\left(\Gamma_{0}(v)\right)$, thus completing the proof of the lemma. For the sake of contradiction, assume that $\left(\pi^{e, v}\right)^{-1}\left(j_{0}\right) \cap \Gamma_{0}(v)=\emptyset$. Moreover, since $e \in E^{\prime},\left(\pi^{e, v}\right)^{-1}\left(j_{0}\right) \cap \Gamma_{1}(v) \leqslant 1$. This yields the following bound:

$$
\left|\sum_{i^{\prime} \in\left(\pi^{e, v}\right)^{-1}\left(j_{0}\right)} \mathbf{b}\left(v, i^{\prime}\right)\right| \leqslant\left(\frac{\tau}{2}+t\left(\frac{\tau}{10 t}\right)\right)\left\|\mathbf{b}_{v}\right\|_{2}=0.6 \tau\left\|\mathbf{b}_{v}\right\|_{2} .
$$


However, the folding constraints (Equation (8)) imply that

$$
\sum_{i \in\left(\pi^{e, u}\right)^{-1}\left(j_{0}\right)} \mathbf{b}(u, i)=\sum_{i^{\prime} \in\left(\pi^{e, v}\right)^{-1}\left(j_{0}\right)} \mathbf{b}\left(v, i^{\prime}\right),
$$

which is a contradiction to Equations (19) and (20) since $\left\|\mathbf{b}_{u}\right\|_{2},\left\|\mathbf{b}_{v}\right\|_{2}>0$ (by the definition of $\left.S_{i r r}\right)$. This completes the proof of the lemma.

We now have all the ingredients to complete the soundness analysis. Let $L^{*}$ be a labeling to the vertices in $S_{i r r}$ constructed by independently and uniformly at random choosing a label from the set $\Gamma_{0}(v)$ for every vertex $v \in S_{i r r}$. By Lemma 4.6, every edge $e=(u, v) \in$ $E^{\prime}$ is satisfied with probability at least $\frac{1}{\left|\Gamma_{0}(u)\right|\left|\Gamma_{0}(v)\right|} \geqslant \frac{\tau^{4}}{16}$ (by Equation (17)). Therefore, in expectation the total fraction $\Delta$ of edges satisfied is bounded by $\Delta \geqslant\left(\frac{\tau^{4}}{16}\right)\left(\theta^{2}-2 \zeta\right)$. Choosing $J>\left(4^{R}\right)^{5}$ and $R \gg 1$ large enough (depending on $\eta$ ) so that $\zeta \ll \theta^{2}$ one can ensure that $\Delta>2^{-c_{0} R}$ thereby yielding a contradiction to the soundness of Theorem 3.5 .

We conclude this section with an observation regarding the instances produced by the hardness reduction. This observation will be useful in Section 5.

LEMMA 4.7. The set of points $\bar{X}$ output by the reduction are in isotropic position within their ambient subspace $F$, that is, for all vectors $\mathbf{b} \in F$,

$$
\mathbb{E}_{\overline{\mathbf{x}} \in \bar{X}}\left[|\langle\mathbf{b}, \overline{\mathbf{x}}\rangle|^{2}\right]=\|\mathbf{b}\|^{2} .
$$

Proof. Let $\Pi_{F}$ denote the projection onto the space $F$. The lemma is an immediate consequence of (6) as shown next.

$$
\begin{aligned}
\mathbb{E}_{\overline{\mathbf{x}} \in \bar{X}}\left[|\langle\mathbf{b}, \overline{\mathbf{x}}\rangle|^{2}\right] & =\mathbb{E}_{v \in V} \mathbb{E}_{\mathbf{x} \in\{-1,1\}^{\mathcal{P}_{v}}}\left[\left\langle\mathbf{b}, \Pi_{F} \mathbf{x}\right\rangle^{2}\right] \\
& =\mathbb{E}_{v \in V} \mathbb{E}_{\mathbf{x} \in\{-1,1\}^{\mathcal{P}_{v}}}\left[\left\langle\Pi_{F} \mathbf{b}, \mathbf{x}\right\rangle^{2}\right] \\
& \left.=\mathbb{E}_{v \in V} \mathbb{E}_{\mathbf{x} \in\{-1,1\}^{\mathcal{P}_{v}}}\left[\left\langle\mathbf{b}_{\mathbf{v}}, \mathbf{x}\right\rangle^{2}\right] \quad \text { (using } \Pi_{F} \mathbf{b}=\mathbf{b}\right) \\
& =\mathbb{E}_{v \in V}\left[\left\|\mathbf{b}_{\mathbf{v}}\right\|^{2}\right]=\|\mathbf{b}\|^{2} . \quad \square
\end{aligned}
$$

\section{HARDNESS REDUCTION FOR QM(P)}

In this section, we shall describe the NP-hardness reduction from the $L_{p}$ subspace minimization problem to the $L_{p}$ Quadratic Grothendieck Maximization Problem $Q M(p)$.

The reduction is carried out in three steps. First, we present a reduction from $L_{p}$ subspace minimization to a variant of $\mathrm{QM}(p)$ where the solution is constrained to be in a subspace, and the diagonal entries of the input are not necessarily zero. In the second step, we will remove the restriction that the solution is constrained in a subspace, and finally reduce it to the case when all the diagonal entries are zero.

To this end, we begin by formally defining the $\mathrm{QM}_{\mathrm{W}}(p)$ optimization problem. The input to $\mathrm{QM}_{\mathrm{W}}(p)$ consists of a symmetric $n \times n$ matrix $A=\left(a_{i j}\right)$ (with possibly nonzero diagonal entries) and a subspace $W \subseteq \mathbb{R}^{n}$ specified by a basis. The goal is to compute the following quantity:

$$
\operatorname{Val}_{\mathrm{p}}^{\mathrm{W}}(A)=\max _{x \in W}\left\{\sum_{i, j=1}^{n} a_{i j} x_{i} x_{j} \mid \sum_{i}\left\|x_{i}\right\|^{p} \leqslant 1\right\} .
$$

We begin by showing the following NP-hardness result for the $\mathrm{QM}_{\mathrm{W}}(p)$ problem. 
Theorem 5.1. For any fixed $p>2$ and $\epsilon>0$, it is $\mathrm{NP}$-hard to approximate the $\mathrm{QM}_{\mathrm{W}}(p)$ problem within a $(1-\epsilon) \gamma_{p}^{2}$ factor even on instances A satisfying

$$
\operatorname{Val}_{p}^{W}(A) \geqslant 1 / \operatorname{dim}(A) \quad \text { and }\|A\| \leqslant \operatorname{dim}(A) .
$$

Proof. We will show that under minor technical conditions, the $L_{p}$ subspace minimization problem with dimension $k=n-1$ reduces to the $\mathrm{QM}_{\mathrm{W}}(p)$ problem.

For the sake of convenience, we restate the $L_{p}$ subspace minimization problem for $k=n-1$. The input is a set of points $\left\{v_{1}, \ldots, v_{m}\right\} \in \mathbb{R}^{n}$. If $x$ denotes the unit normal vector to the unknown subspace, then the goal is to

$$
\operatorname{minimize}\left(\sum_{i}\left\langle v_{i}, x\right\rangle^{p}\right)^{1 / p} \quad \text { subject to } \quad\left(\sum_{i} x_{i}^{2}\right)^{1 / 2}=1 .
$$

We can rewrite the $L_{p}$ subspace minimization problem as a quadratic maximization by switching the constraint and the objective function. Specifically, we can write

$$
\text { maximize }\left(\sum_{i \in[n]} y_{i}^{2}\right) \quad \text { subject to } \quad\left(\sum_{i \in[m]}\left\langle v_{i}, y\right\rangle^{p}\right)^{1 / p} \leqslant 1 \text {. }
$$

It is easy to see that the preceding formulation is equivalent to $L_{p}$ subspace minimization. Given a solution $x \in \mathbb{R}^{n}$ for (21) with objective value $c, y=x / c$ has objective value $\frac{1}{c^{2}}$ for (22). Conversely, if $y \in \mathbb{R}^{n}$ has an objective value $\frac{1}{c^{2}}$ on (22), then $x=c y$ is a feasible solution to (21) with objective value $c$.

Let $M$ denote the $m \times n$ matrix whose rows are the vectors $\left\{v_{i}\right\}$. If $\operatorname{rank}(M)<n$, then there exists a vector $x$ such that $M x=0$, that is, all the points $\left\{v_{j}\right\}_{j \in[m]}$ lie on the hyperplane defined by $\langle x, v\rangle=0$. We can compute $x$ with $M x=0$ by solving a system of linear equations, and output the solution $x$ with cost zero.

If $\operatorname{rank}(M)=n$, then fix $A=M\left(M^{T} M\right)^{-2} M^{T}$. Let $W \subset \mathbb{R}^{m}$ denote the subspace spanned by the columns of $M$. For every $y \in \mathbb{R}^{n}$, if $z=M y$, then $z^{T} A z=$ $y^{T} M^{T}\left(M\left(M^{T} M\right)^{-2} M^{T}\right) M y=y^{T} y$. Hence, we can rewrite $(22)$ to obtain the $\mathrm{QM}_{\mathrm{W}}(p)$ problem with the variables $z=M y$. More precisely, we can rewrite (22) as follows:

$$
\text { maximize } z^{T} A z \quad \text { subject to } \sum_{i \in[m]} z_{i}^{p} \leqslant 1 \text { and } z \in W .
$$

By Theorem 1.1, for every $\epsilon>0$, it is NP-hard to approximate the $L_{p}$ subspace minimization problem to within a $(1-\epsilon) \gamma_{p}$ factor. Therefore, it is NP-hard to approximate the $\mathrm{QM}_{\mathrm{W}}(p)$ problem to a ratio of $(1-\epsilon) \gamma_{p}^{2}$.

Finally, we need to check that the instance $A$ satisfies the desired bounds on $\operatorname{Val}_{p}^{W}(A)$ and $\|A\| \cdot \operatorname{Val}_{\underline{p}}^{W}(A)$ is the optimum of (23), which is equal to the optimum of (22). Since $y=\frac{1}{m^{1 / p}} \cdot \frac{1}{\sqrt{n}} \overrightarrow{1}$ is a feasible solution for $(22)$, we conclude that $\operatorname{Val}_{p}^{W}(A) \geqslant \frac{1}{m^{2 / p}}$. Further, using Lemma 4.7 we can assume that the vectors $\left\{v_{i}\right\}_{i \in[m]}$ are in isotropic position. This implies that $M^{T} M=\frac{m}{n} \cdot I$ and the spectral norm of $A$ is at most

$$
\|A\| \leqslant\|M\|\left\|\left(M^{T} M\right)^{-1}\right\|^{2}\left\|M^{T}\right\| \leqslant\|M\|_{F r}\left\|\left(M^{T} M\right)^{-1}\right\|^{2}\left\|M^{T}\right\|_{F r} \leqslant \sqrt{m} \cdot\left(\frac{n}{m}\right)^{2} \cdot \sqrt{m}<m,
$$

where $\|M\|_{F r}$ denotes the Frobenius norm of the matrix $M$. 


\subsection{Removing the Subspace Constraint}

In the second step of our reduction, we will present a reduction from $\mathrm{QM}_{\mathrm{W}}(p)$ to $\mathrm{QM}(p)$ where the solution can be an arbitrary vector in $\mathbb{R}^{n}$.

LEMma 5.2. There is a polynomial time algorithm that given a matrix $n \times n$-matrix $A=\left(a_{i j}\right)$, a subspace $W \subseteq \mathbb{R}^{n}$, and $\epsilon>0$, produces a $n \times n$ matrix $C=\left(c_{i j}\right)$ such that

$$
\frac{\operatorname{Val}_{p}(C)}{\operatorname{Val}_{p}^{W}(A)} \in[1,1+\epsilon]
$$

and

$$
\|C\| \leqslant \frac{200 n^{3}\|A\|^{3}}{\epsilon \operatorname{Val}_{p}^{W}(A)} .
$$

Proof. Let $\Pi_{W^{\perp}}$ denote the projection onto the orthogonal complement of the vector space $W$. Fix $C=A-(\|A\| / \eta) \cdot \Pi_{W^{\perp}}$ where $\eta$ is a parameter whose value will be fixed to $\eta:=\epsilon^{2}\left(\operatorname{Val}_{p}^{W}(A)\right)^{2} / 100 n^{3}\|A\|^{2}$. It is easy to check that the matrix $C$ can be efficiently generated given $A$ and a basis for $W$ and satisfies the desired bound on $\|C\|$.

For every $x \in W, x^{T} C x=x^{T} A x-\|A\| / \eta \cdot\left\|\Pi_{W^{\perp}} x\right\|^{2}=x^{T} A x$. This implies that $\operatorname{Val}_{p}(C) \geqslant \operatorname{Val}_{p}^{W}(A)$.

Suppose $x \in \mathbb{R}^{n}$ to be the vector that achieves the maximum corresponding to $\operatorname{Val}_{p}(C)$, that is, $\operatorname{Val}_{p}(C)=x^{T} C x$ and $\|x\|_{p} \leqslant 1$. Since $\operatorname{Val}_{p}(C) \geqslant 0$, we will have

$$
\left\|\Pi_{W^{\perp}} x\right\|^{2} \leqslant \frac{x^{T} A x}{\|A\| / \eta} \leqslant \eta\|x\|^{2} \leqslant \eta\left(n^{1-2 / p}\|x\|_{p}^{2}\right) \leqslant n \eta .
$$

This implies that

$$
\left\|\Pi_{W^{\perp}} x\right\|_{p} \leqslant\left\|\Pi_{W^{\perp}} x\right\|_{2} \leqslant(n \eta)^{1 / 2} .
$$

If we let $y=\Pi_{W} x /\left\|\Pi_{W} x\right\|_{p}$, then clearly $y \in W$ and $\|y\|_{p} \leqslant 1$. Using the triangle inequality, we have $\left\|\Pi_{W} x\right\|_{p} \leqslant\|x\|_{p}+\left\|\Pi_{W^{\perp}} x\right\|_{p} \leqslant 1+\eta$. Furthermore,

$$
\begin{aligned}
y^{T} A y & =\frac{1}{\left\|\Pi_{W} x\right\|_{p}^{2}}\left(x^{T} A x-2 x^{T} A \Pi_{W^{\perp}} x+x \Pi_{W^{\perp}} A \Pi_{W^{\perp}} x\right) \\
& \geqslant \frac{1}{\left\|\Pi_{w} x\right\|_{p}^{2}}\left(x^{T} A x-2\|A\|\|x\|\left\|\Pi_{W^{\perp}} x\right\|-\|A\|\left\|\Pi_{W^{\perp}} x\right\|^{2}\right) \\
& \geqslant \frac{1}{\left\|\Pi_{w} x\right\|_{p}^{2}}\left(x^{T} A x-2 n^{1 / 2-1 / p}\|A\|\|x\|_{p}\left\|\Pi_{W^{\perp}} x\right\|-\|A\|\left\|\Pi_{W^{\perp}} x\right\|^{2}\right) \\
& \geqslant \frac{1}{\left\|\Pi_{w} x\right\|_{p}^{2}}\left(\operatorname{Val}_{p}(C)-2 n^{1 / 2-1 / p}\|A\|\|x\|_{p}\left\|\Pi_{W^{\perp}} x\right\|-\|A\|\left\|\Pi_{W^{\perp}} x\right\|^{2}\right) \\
& \geqslant(1-\epsilon) \operatorname{Val}_{p}(C),
\end{aligned}
$$

where the last step uses (24) and the choice of $\eta$. Since $\operatorname{Val}_{p}^{W}(A) \geqslant y^{T} A y$, the desired result follows.

\subsection{Removing the Diagonal Entries}

In this final step of the reduction, we will create an instance of the problem with zeros on the diagonal. The idea is to replicate all the coordinates a large number $(D)$ times to ensure that the contribution of the diagonal entries to the objective is negligible and thus the diagonal terms can be removed. 
Lemma 5.3. There is an algorithm that given a matrix Aand an $\epsilon>0$, finds a matrix $B$ that has zero on its diagonal entries and $\operatorname{Val}_{p}(B) / \operatorname{Val}_{p}(A) \in[1-\epsilon, 1]$. The running time of the algorithm is a polynomial in $n, \frac{1}{\epsilon}$ and the parameter $\beta=\|A\| / \lambda(A)$ where $\lambda(A)$ is the largest positive eigenvalue of $A$.

Proof. Suppose $\lambda$ is the largest eigenvalue of $A$. If $\lambda \leqslant 0$, then $\operatorname{Val}_{p}(A)=0$, which implies that $B=0$ satisfies the assertion of the lemma.

Suppose $x$ is an eigenvector with eigenvalue $\lambda$, then $y:=\frac{x}{\|x\|_{p}}$ satisfies $\|y\|_{p} \leqslant 1$ and $y^{T} A y=\lambda \cdot\|x\|^{2} /\|x\|_{p}^{2} \geqslant \lambda / n^{2-2 / p}$. Therefore, we have

$$
\operatorname{Val}_{p}(A)>\lambda(A) / n^{2-2 / p} .
$$

The matrix $B$ will be a $n D \times n D$ matrix for $D=\left(n^{3} \beta / \epsilon\right)^{p /(p-2)}$. The matrix $B$ will have a $D \times D$ block $B_{i j}=\left(b_{i j}^{(k \ell)}\right)$ for each entry $a_{i j}$ of $A$. Set $M:=D^{1-1 / p}$. The entries are given by

$$
b_{i j}^{(k \ell)}:= \begin{cases}0 & \text { if } i=j \text { and } k=\ell \\ a_{i j} / M^{2} & \text { otherwise. }\end{cases}
$$

Suppose $x \in \mathbb{R}^{n D}$ achieves the maximum corresponding to $\operatorname{Val}_{p}(B)$. Define $y \in \mathbb{R}^{n}$ by setting $y_{i}=\frac{1}{M} \sum_{k \in[D]} x_{i}^{(k)}$. The $p$-norm of $y$ can be bounded by

$$
\|y\|_{p}^{p}=\sum_{i \in[n]}\left(\frac{1}{M} \sum_{k \in[D]} x_{i}^{(k)}\right)^{p} \leqslant \frac{D^{p-1}}{M^{p}} \cdot \sum_{i \in[n], k \in[D]}\left(x_{i}^{(k)}\right)^{p}=\|x\|_{p}^{p}=1 .
$$

We can compare $x^{T} B x$ with $y^{T} A y$ as follows:

$$
\begin{aligned}
\operatorname{Val}_{p}(B)=x^{T} B x & =\frac{1}{M^{2}} \sum_{i, j \in[n]} \sum_{k, \ell \in[D]} a_{i j} x_{i}^{(k)} x_{j}^{(\ell)}-\frac{1}{M^{2}} \sum_{i \in[n], k \in[D]} a_{i i}\left(x_{i}^{(k)}\right)^{2} \\
& =\sum_{i, j \in[n]} a_{i j}\left(\frac{1}{M} \sum_{k \in[D]} x_{i}^{(k)}\right)\left(\frac{1}{M} \sum_{\ell \in[D]} x_{j}^{(\ell)}\right)-\frac{1}{M^{2}} \sum_{i \in[n], k \in[D]} a_{i i} \cdot\left(x_{i}^{(k)}\right)^{2} \\
& =\sum_{i, j \in[n]} a_{i j} y_{i} y_{j}-\frac{1}{M^{2}} \sum_{i \in[n], k \in[D]} a_{i i} \cdot\left(x_{i}^{(k)}\right)^{2} \\
& =y^{T} A y-\frac{1}{M^{2}} \sum_{i \in[n], k \in[D]} a_{i i}\left(x_{i}^{(k)}\right)^{2} \\
& \leqslant \operatorname{Val}_{p}(A)+\frac{n D\|A\|}{M^{2}}\left(\mathrm{using} \max _{i}\left|a_{i i}\right| \leqslant\|A\| \text { and }\left|x_{i}^{(k)}\right| \leqslant 1\right) \\
& \leqslant \operatorname{Val}_{p}(A)\left(1+\frac{n^{3-2 / p}\|A\|}{\lambda(A) D^{1-2 / p}}\right) \quad(\mathrm{using}(25)) \\
& \leqslant \operatorname{Val}_{p}(A)(1+\epsilon), \quad
\end{aligned}
$$

where in the final step we used the choice of $D$.

Conversely, suppose $y \in \mathbb{R}^{n}$ achieves the maximum corresponding to $\operatorname{Val}_{p}(A)$. Define $x \in \mathbb{R}^{n D}$ by setting $x_{i}^{(k)}=D^{-1 / p} y_{i}$. Clearly, $\|x\|^{p}=\sum_{i \in[n], k \in[D]}\left(x_{i}^{(k)}\right)^{p}=\sum_{i \in[n]} y_{i}^{p}=\|y\|_{p}^{p}$. 
Furthermore,

$$
\begin{aligned}
\operatorname{Val}_{p}(B) & \geqslant x^{T} B x \\
& =\sum_{i, j \in[n]} a_{i j}\left(\frac{1}{M} \sum_{k \in[D]} x_{i}^{(k)}\right)\left(\frac{1}{M} \sum_{\ell \in[D]} x_{j}^{(\ell)}\right)-\frac{1}{M^{2}} \sum_{i \in[n], k \in[D]} a_{i i}\left(x_{i}^{(k)}\right)^{2} \\
& =\sum_{i, j \in[n]} a_{i j} y_{i} y_{j}-\frac{1}{D} \sum_{i \in[n]} a_{i i} y_{i}^{2} \\
& \geqslant \operatorname{Val}_{p}(A)-\frac{n}{D} \max _{i}\left|a_{i i}\right| \\
& \geqslant \operatorname{Val}_{p}(A)\left(1-\frac{n^{3-2 / p}\|A\|}{D \lambda(A)}\right)
\end{aligned}
$$

where in the final step we used that $y_{i}^{2}<1$ for all $i \in[n]$, and inequality (25). From (27) and (28) and the choice of $D$, the result follows.

We have all the ingredients to wrap up the proof of Theorem 1.2.

Proof. The idea is to apply the reductions in Theorem 5.1, Lemma 5.2, and Lemma 5.3 sequentially. The running time of the third step of the reduction depends polynomially on the ratio $\beta=\|A\| / \lambda(A)$. By $(25), \beta<n\|A\| / \operatorname{Val}_{p}(A)$. In Theorem 5.1, the instances produced have $\|A\|$ and $\operatorname{Val}_{p}(A)$ bounded polynomially in dimension. The reduction in Lemma 5.2 preserves this property by virtue of the bounds on $\|C\|$ and $\operatorname{Val}_{p}(C)$ for the output instance $C$.

\section{APPROXIMATION FOR QM(P)}

Let $A=\left(a_{i j}\right)_{i, j=1}^{n}$ be an $n \times n$ symmetric matrix with diagonal entries all zero, given as an instance of $\mathrm{QM}(p)$ for a fixed $p \geqslant 2$. We have

$$
\operatorname{Val}_{p}(A)=\max \left\{\sum_{i, j=1}^{n} a_{i j} x_{i} x_{j}:\left\{x_{1}, \ldots, x_{n}\right\} \subseteq \mathbb{R}, \sum_{i=1}^{n}\left|x_{i}\right|^{p} \leqslant 1\right\} .
$$

The preceding can be relaxed to the following optimization over vectors,

$$
\operatorname{Vec}_{p}(A)=\max \left\{\sum_{i, j=1}^{n} a_{i j}\left\langle u_{i}, u_{j}\right\rangle:\left\{u_{1}, \ldots, u_{n}\right\} \subseteq L_{2}, \sum_{i=1}^{n}\left\|u_{i}\right\|_{2}^{p} \leqslant 1\right\} .
$$

As shown in Kindler et al. [2010], the optimization problem $\operatorname{Vec}_{p}(A)$ is a convex program for $p \geqslant 2$, and can be solved in polynomial time to arbitrary precision. Using this, we shall prove the following theorem, which implies Theorem 1.3.

Theorem 6.1. For any fixed $p \geqslant 2, \operatorname{Vec}_{p}(A) \leqslant \gamma_{p}^{2} \cdot \operatorname{Val}_{p}(A)$ for any instance $A$ of $\mathrm{QM}(p)$. This implies a polynomial time $\gamma_{p}^{2}$ approximation for $\mathrm{QM}(p)$.

Furthermore, for all constants $\epsilon>0$, there is a polynomial time (randomized) rounding procedure that rounds the solution to $\operatorname{Vec}_{p}(A)$ to obtain a $(1+\epsilon) \gamma_{p}^{2}$ approximate solution to $\operatorname{Val}_{p}(A)$.

The remainder of this section is devoted to proving the preceding theorem. Let $v_{1}, \ldots, v_{n}$ denote an optimal solution to the preceding convex program. Let $h_{1}, \ldots, h_{n}$ be mean zero Gaussian random variables obtained by defining $h_{i}:=\left\langle G, v_{i}\right\rangle(1 \leqslant i \leqslant n)$, where $G$ 
is a random Gaussian vector in the space spanned by $v_{1}, \ldots, v_{n}$. It is easy to see that the following properties are satisfied.

$$
\mathbb{E}\left[\sum_{i, j=1}^{n} a_{i j} h_{i} h_{j}\right]=\operatorname{Vec}_{p}(A) \text { and } \sum_{i=1}^{n}\left(\mathbb{E}\left[h_{i}^{2}\right]\right)^{\frac{p}{2}} \leqslant 1 .
$$

Now we simply note that

$$
\begin{aligned}
\operatorname{Vec}_{p}(A) & =\mathbb{E}\left[\sum_{i, j=1}^{n} a_{i j} h_{i} h_{j}\right] \\
& =\mathbb{E}\left[\left(\sum_{k=1}^{n}\left|h_{k}\right|^{p}\right)^{2 / p}\left[\sum_{i, j=1} a_{i j}\left(\frac{h_{i}}{\left(\sum_{k=1}^{n}\left|h_{k}\right|^{p}\right)^{1 / p}}\right) \cdot\left(\frac{h_{j}}{\left(\sum_{k=1}^{n}\left|h_{k}\right|^{p}\right)^{1 / p}}\right)\right]\right] .
\end{aligned}
$$

Applying the definition of $\operatorname{Val}_{p}(A)$ we obtain

$$
\begin{aligned}
\operatorname{Vec}_{p}(A) & \leqslant \mathbb{E}\left[\left(\sum_{k=1}^{n}\left|h_{k}\right|^{p}\right)^{2 / p} \cdot \operatorname{Val}_{p}(A)\right] \\
& \leqslant\left(\sum_{k=1}^{n} \mathbb{E}\left[\left|h_{k}\right|^{p}\right]\right)^{2 / p} \operatorname{Val}_{p}(A) \quad \text { (by Jensen's inequality and since } p \geqslant 2 \text { ) } \\
& =\left(\sum_{k=1}^{n} \gamma_{p}^{p}\left(\mathbb{E}\left[h_{k}^{2}\right]\right)^{p / 2}\right)^{2 / p} \operatorname{Val}_{p}(A),
\end{aligned}
$$

where the last step used the fact that $h_{k}$ is a Gaussian random variable with mean zero, and the definition of $\gamma_{p}$. Therefore, by Equation $(29), \operatorname{Vec}_{p}(A) \leqslant \gamma_{p}^{2} \operatorname{Val}_{p}(A)$, which is the desired upper bound. Note that this bound is obtained directly without rounding the vectors. To complete the proof of Theorem 6.1 we need to demonstrate a polynomial time rounding algorithm that extracts a $\gamma_{p}^{2}(1+\delta)$ approximate solution $x_{1}^{*}, \ldots, x_{n}^{*}$ to $\operatorname{Val}_{p}(A)$ from the vectors $v_{1}, \ldots, v_{n}$ for any constant $\delta>0$. This shall be our goal in the remainder of the section.

Before we do so we can first assume without the loss of generality that

$$
\left|a_{12}\right|=1=\max _{1 \leqslant i, j \leqslant n}\left|a_{i j}\right|,
$$

by appropriately relabeling the entries of the matrix $A$ and scaling them. Setting $x_{1}=1 / 2$ and $x_{2}=a_{12} /\left(2\left|a_{12}\right|\right)$ and $x_{3}, \ldots, x_{n}=0$ we obtain that

$$
\operatorname{Vec}_{p}(A) \geqslant \operatorname{Val}_{p}(A) \geqslant 1 / 4 \text {. }
$$

The following is the rounding algorithm that we shall analyze.

$\operatorname{Algorithm} \operatorname{Round}\left(A,\left\{v_{1}, \ldots, v_{n}\right\}\right)$ :

(1) Let $T:=n^{22}$. Sample $T$ random Gaussian vectors $G_{1}, \ldots, G_{T}$ in the span of $v_{1}, \ldots, v_{n}$.

(2) Define random variables $z_{i}^{(t)}:=\left\langle G_{t}, v_{i}\right\rangle$ for all $1 \leqslant t \leqslant T$ and $1 \leqslant i \leqslant n$. In addition, define

$$
x_{i}^{(t)}:=\frac{z_{i}^{(t)}}{\left(\sum_{j=1}^{n}\left|z_{j}^{(t)}\right|^{p}\right)^{1 / p}} \quad \text { and } \quad \Delta_{t}:=\sum_{i, j=1}^{n} a_{i j} x_{i}^{(t)} x_{j}^{(t)}
$$


(3) Let $t^{*} \in\{1, \ldots, T\}$ be such that $\Delta_{t^{*}}=\max _{1 \leqslant t \leqslant T} \Delta_{t}$. Output $x_{1}^{*}, \ldots, x_{n}^{*}$ as the solution where $x_{i}^{*}=x_{i}^{\left(t^{*}\right)}$ for $1 \leqslant i \leqslant n$.

Let $\mathbb{E}_{t}$ denote the expectation over the uniformly at random choice of $t$ from $1, \ldots, T$. We begin with the following lemma.

Lemma 6.2. Given the random variables constructed in the procedure $\operatorname{Round}(A$, $\left.\left\{v_{1}, \ldots, v_{n}\right\}\right)$, with probability at least $1-1 / n^{8}$ over the choice of $G_{1}, \ldots, G_{T}$ the following inequality holds:

$$
\sum_{i, j=1}^{n} a_{i j} \mathbb{E}_{t}\left[z_{i}^{(t)} z_{j}^{(t)}\right] \geqslant\left(1-8 / n^{2}\right) \operatorname{Vec}_{p}(A) .
$$

Proof. We begin by ignoring the terms corresponding to pairs $i, j(1 \leqslant i, j \leqslant n)$ such that $\left\langle v_{i}, v_{j}\right\rangle$ is very small. Formally, Let $R:=\left\{(i, j) \in[n] \times[n] \quad\left|\left\langle v_{i}, v_{j}\right\rangle\right| \geqslant 1 / n^{4}\right\}$. We have

$$
\begin{aligned}
\left|\sum_{(i, j) \in R} a_{i j}\left\langle v_{i}, v_{j}\right\rangle-\sum_{i, j=1}^{n} a_{i j}\left\langle v_{i}, v_{j}\right\rangle\right| & \leqslant \sum_{(i, j) \notin R}\left|a_{i j}\left\langle v_{i}, v_{j}\right\rangle\right| \\
& \leqslant \sum_{(i, j) \notin R}\left|a_{i j}\right|\left(\frac{1}{n^{4}}\right) \\
& \leqslant n^{2}\left(\frac{1}{n^{4}}\right)=\frac{1}{n^{2}} . \quad \text { (by Equation (30)). }
\end{aligned}
$$

Now consider any $(i, j) \in R$. As before, we have Gaussian random variables $h_{i}$ and $h_{j}$ such that $\mathbb{E}\left[h_{i} h_{j}\right]=\left\langle v_{i}, v_{j}\right\rangle$. Moreover, since $(i, j) \in R,\left|\mathbb{E}\left[h_{i} h_{j}\right]\right| \geqslant 1 / n^{4}$. We also need a bound on the variance of $h_{i} h_{j}$. Clearly, $\operatorname{Var}\left[h_{i} h_{j}\right] \leqslant \mathbb{E}\left[h_{i}^{2} h_{j}^{2}\right]$. Also, from Equation (29) we have that $\mathbb{E}\left[h_{i}^{2}\right], \mathbb{E}\left[h_{j}^{2}\right] \leqslant 1$. Therefore, $\mathbb{E}\left[h_{i}^{2} h_{j}^{2}\right]$ is upper bounded by $\mathbb{E}\left[g^{4}\right]=3$ where $g$ is a standard Gaussian variable with variance 1 . We note that over the choice of $G_{1}, \ldots, G_{T}$, the random variables $z_{i}^{(t)} z_{j}^{(t)}$ are identically distributed as $h_{i} h_{j}$ for all $1 \leqslant t \leqslant T$. Moreover, since $G_{1}, \ldots, G_{T}$ are independent Gaussian vectors, the random variables $z_{i}^{(t)} z_{j}^{(t)}$ are also independent for $1 \leqslant t \leqslant T$. Therefore,

$$
\operatorname{Var}\left[\mathbb{E}_{t}\left[z_{i}^{(t)} z_{j}^{(t)}\right]\right] \leqslant \frac{\operatorname{Var}\left[h_{i} h_{j}\right]}{T} \leqslant \frac{1}{n^{20}}
$$

by our choice of $T$ and where the variance is over the choice of $G_{1}, \ldots, G_{T}$. Moreover, since $\mathbb{E}\left[\mathbb{E}_{t}\left[z_{i}^{(t)} z_{j}^{(t)}\right]\right]=\mathbb{E}\left[h_{i} h_{j}\right]$, we have the following bound using Chebyshev's inequality.

$$
\operatorname{Pr}\left[\left|\mathbb{E}_{t}\left[z_{i}^{(t)} z_{j}^{(t)}\right]-\mathbb{E}\left[h_{i} h_{j}\right]\right| \geqslant 1 / n^{5}\right] \leqslant 1 / n^{10}
$$

Since the preceding analysis holds for all pairs $(i, j) \in R$, using a union bound over all pairs the preceding implies that with probability at least $1-1 / n^{8}$, the following holds:

$$
\left|\sum_{(i, j) \in R} a_{i j} \mathbb{E}_{t}\left[z_{i}^{(t)} z_{j}^{(t)}\right]-\sum_{(i, j) \in R} a_{i j} \mathbb{E}\left[h_{i} h_{j}\right]\right| \leqslant\left(1 / n^{5}\right) \sum_{(i, j) \in R} a_{i j} \leqslant 1 / n^{3},
$$


where the final inequality is obtained using Equation (30). Combining the preceding with Equation (33) implies that with probability at least $1-1 / n^{8}$, the following holds:

$$
\left|\sum_{i, j=1}^{n} a_{i j} \mathbb{E}_{t}\left[z_{i}^{(t)} z_{j}^{(t)}\right]-\sum_{i, j=1}^{n} a_{i j}\left\langle v_{i}, v_{j}\right\rangle\right| \leqslant 1 / n^{2}+1 / n^{3} \leqslant 2 / n^{2} .
$$

This implies that with probability at least $1-1 / n^{8}$,

$$
\sum_{i, j=1}^{n} a_{i j} \mathbb{E}_{t}\left[z_{i}^{(t)} z_{j}^{(t)}\right] \geqslant \operatorname{Vec}_{p}(A)-2 / n^{2} \geqslant\left(1-8 / n^{2}\right) \operatorname{Vec}_{p}(A)
$$

where the last inequality follows from Equation (31). This completes the proof of the lemma.

The next lemma also proves a similar bound for the pth moments of the variables Gaussian variables $h_{i}$.

Lemma 6.3. With probability at least $1-1 / n^{8}$ over the choice of $G_{1}, \ldots, G_{T}$ the following holds for every $i=1, \ldots, n$.

$$
\left|\mathbb{E}_{t}\left[\left|z_{i}^{(t)}\right|^{p}\right]-\mathbb{E}\left[\left|h_{i}\right|^{p}\right]\right| \leqslant 1 / n^{4}
$$

Proof. Let us fix $i \in\{1, \ldots, n\}$ for the moment. As noted before, over the choice of $G_{1}, \ldots, G_{T}$ the random variables $z_{i}^{(t)}, 1 \leqslant t \leqslant T$, are independent random variables distributed identically to $h_{i}$, which is a mean zero Gaussian random variable that satisfies

$$
\operatorname{Var}\left[\left|h_{i}\right|^{p}\right] \leqslant \mathbb{E}\left[\left|h_{i}\right|^{2 p}\right] \leqslant \gamma_{2 p}^{2 p}\left(\mathbb{E}\left[\left|h_{i}^{2}\right|\right]\right)^{p} \leqslant \gamma_{2 p}^{2 p},
$$

where the second to last inequality is by the definition of $\gamma_{2 p}$ and the last inequality uses Equation (29). Since $z_{i}^{(t)}$ are independent for $1 \leqslant t \leqslant T$, this implies

$$
\operatorname{Var}\left[\mathbb{E}_{t}\left[\left|z_{i}^{(t)}\right|^{p}\right]\right] \leqslant \frac{\operatorname{Var}\left[\left|h_{i}\right|^{p}\right]}{T} \leqslant \frac{\gamma_{2 p}^{2 p}}{n^{22}} \leqslant \frac{1}{n^{20}}
$$

for large enough $n$. Therefore, by Chebyshev's inequality we obtain

$$
\operatorname{Pr}\left[\left|\mathbb{E}_{t}\left[\left|z_{i}^{(t)}\right|^{p}\right]-\mathbb{E}\left[\left|h_{i}\right|^{p}\right]\right| \geqslant 1 / n^{5}\right] \leqslant 1 / n^{10}
$$

Taking a union bound over all $i=1, \ldots, n$ and rearranging Equation (39) proves the lemma.

We are now ready to prove the desired bounds on the performance of the rounding algorithm $\operatorname{Round}\left(A,\left\{v_{1}, \ldots, v_{n}\right\}\right)$. For this we need to prove an upper bound on $\operatorname{Vec}_{p}(A)$ in terms of $\Delta^{*}$. This is shown through the following series of inequalities implied by 
the two previous lemmas whose conditions hold with probability at least $1-2 / n^{8}$.

$$
\begin{aligned}
& \left(1-8 / n^{2}\right) \operatorname{Vec}_{p}(A) \\
& \leqslant \mathbb{E}_{t}\left[\sum_{i, j=1}^{n} a_{i j} z_{i}^{(t)} z_{j}^{(t)}\right] \quad \text { (by Lemma 6.2) } \\
& \quad \leqslant \mathbb{E}_{t}\left[\left(\sum_{k=1}^{n}\left|z_{k}^{(t)}\right|^{p}\right)^{2 / p} \cdot\left(\sum_{i, j=1}^{n} a_{i j}\left(\frac{z_{i}^{(t)}}{\left(\sum_{k=1}^{n}\left|z_{k}^{(t)}\right|^{p}\right)^{1 / p}}\right) \cdot\left(\frac{z_{j}^{(t)}}{\left(\sum_{k=1}^{n}\left|z_{k}^{(t)}\right|^{p}\right)^{1 / p}}\right)\right)\right] \\
& \left.\leqslant \mathbb{E}_{t}\left[\left(\sum_{k=1}^{n}\left|z_{k}^{(t)}\right|^{p}\right)^{2 / p} \Delta^{*}\right] \quad \text { (by definition of } \Delta^{*}\right) \\
& \leqslant\left(\sum_{k=1}^{n} \mathbb{E}_{t}\left[\left|z_{k}^{(t)}\right|^{p}\right]\right)^{2 / p} \Delta^{*} \quad \text { (by Jensen's inequality). }
\end{aligned}
$$

Using the preceding along with Lemma 6.3 and the definition of $\gamma_{p}$ we obtain

$$
\begin{aligned}
& \left(1-8 / n^{2}\right) \operatorname{Vec}_{p}(A) \\
& \quad \leqslant\left(\sum_{k=1}^{n}\left[\mathbb{E}\left[\left|h_{k}\right|^{p}\right]+1 / n^{4}\right]\right)^{2 / p} \Delta^{*} \\
& \quad=\left(\sum_{k=1}^{n}\left[\gamma_{p}^{p}\left(\mathbb{E}\left[\left|h_{k}\right|^{2}\right]\right)^{p / 2}+1 / n^{4}\right]\right)^{2 / p} \Delta^{*} \quad \text { (since } h_{k} \text { is a mean zero Gaussian) } \\
& \left.\leqslant \gamma_{p}^{2}\left(1+1 / n^{3}\right)^{2 / p} \Delta^{*} \quad \text { (by Equation }(29)\right) .
\end{aligned}
$$

Since the parameter $n$ is large enough, the preceding analysis proves the approximation achieved by the rounding algorithm. This completes the proof of Theorem 6.1 and concludes this section.

\section{APPENDIX}

\section{A. CONSTRUCTION OF SMOOTH LABEL COVER AND PROOF OF THEOREM 3.5}

An instance $\phi$ of Max-3-SAT(5) is a 3-CNF formula in which each variable occurs in exactly five clauses. The PCP Theorem [Arora and Safra 1998; Arora et al. 1998] states that it is NP-hard to decide whether $\phi$ is satisfiable (YES instance), or at most $1-\epsilon_{0}$ fraction of its clauses can be satisfied by any assignment, for some universal constant $\epsilon_{0}>0$ (NO instance).

Consider the following reduction from Max-3-SAT(5) to a 2-Prover 1-Round Game. Given an instance $\phi$ of Max-3-SAT(5), the verifier chooses a subset $\mathcal{C}$ of $(J+1) R$ clauses at random, and a subset $\mathcal{C}^{\prime}$ of $R$ clauses from $\mathcal{C}$. Independently for each clause in $\mathcal{C}^{\prime}$, the verifier chooses a variable uniformly at random from those in the clause. Let the collection of these variables be $\mathcal{X}$. The verifier sends the set $\mathcal{C}$ of clauses to the first prover, and the sets $\mathcal{C} \backslash \mathcal{C}^{\prime}$ of clauses and $\mathcal{X}$ of variables to the second prover. The verifier expects back from each prover an assignment to all the variables received by it, and accepts if the answers of the provers are consistent on the common variables and satisfy all the clauses in $\mathcal{C}$. 
The preceding construction is due to Khot [2002a], and is equivalent to a bipartite Label Cover instance $\mathcal{B}$ on vertex sets $U$ and $V$, with projections $\pi_{v u}:[M] \rightarrow[N]$ for edges between $u \in U$ and $v \in V$, where $M=7^{(J+1) R}$ and $N=2^{R} 7^{J R}$. The graph is biregular with the degree of vertices in $U$ being $5^{R}$, and of vertices in $V$ being $\left(\begin{array}{c}(J+1) R \\ R\end{array}\right) 3^{R}$. For each $i \in[N]$ and edge $(u, v),\left|\pi_{v u}^{-1}(i)\right| \leqslant t:=4^{R}$. The size of the construction is $n^{O(J R)}$, where $n$ is the number of clauses in $\phi$. A pair of labelings $\sigma_{U}: U \rightarrow[N]$ and $\sigma_{V}: V \rightarrow[M]$ satisfy an edge $(u, v)$ if $\sigma_{U}(u)=\pi_{v u}\left(\sigma_{V}(v)\right)$.

Consider an $R$-round parallel repetition of the standard clause-variable 2-Prover 1-Round game derived from $\phi$. It is shown formally in Khot [2002a] that this game can be embedded into $\mathcal{L}$. Therefore, the PCP Theorem and the Parallel Repetition Theorem [Raz 1998] yield a universal constant $c_{0}>0$ such that,

-(YES Case) if $\phi$ is a YES instance, then there is a labeling to $U$ and $V$ that satisfies all the edges of $\mathcal{B}$;

- (NO Case) if $\phi$ is a NO instance, then every labeling to $U$ and $V$ satisfies less than a fraction $2^{-c_{0} R}$ fraction of edges of $\mathcal{B}$.

Furthermore, as Khot [2002a] shows, the instance $\mathcal{B}$ satisfies the following smoothness property: for any vertex $w \in V$ and $i, j \in[M]$ such that $i \neq j$,

$$
\mathbf{P r}_{u \sim w}\left[\pi_{w u}(i)=\pi_{w u}(j)\right] \leqslant 1 / J,
$$

where the probability is taken over a random neighbor $u$ of $w$. This is because $i$ and $j$ correspond to distinct assignments to a fixed set of clauses $\mathcal{C}$ (received by first prover) differing on at least one clause, say $C \in \mathcal{C}$. The left-hand side of the preceding equation is upper bounded by the probability (over the choice of $\mathcal{C}^{\prime}$ ) that $C \in \mathcal{C}^{\prime}$, which is at most $1 / J$.

The preceding instance is converted into an instance $\mathcal{L}$ of Smooth Label Cover with vertex set $V$ and label sets $[M]$ and $[N]$ as follows: for every vertex $u \in U$ and its neighbors $v$ and $w$, add an edge $e=\{v, w\}$ in $\mathcal{L}$ with projections $\pi^{e, v}=\pi_{v u}$ and $\pi^{e, w}=\pi_{w u}$. The biregularity and smoothness of $\mathcal{B}$ along with this construction directly imply that $\mathcal{L}$ is regular with degree depending only on $R$ and $J$, and satisfies the smoothness property in Theorem 3.5.

Given labelings $\sigma_{U}$ and $\sigma_{V}$ that satisfy all edges in $\mathcal{B}, \sigma_{V}$ satisfies all edges in $\mathcal{L}$. Thus, a YES instance $\mathcal{B}$ is transformed into a YES instance $\mathcal{L}$. On the other hand, assume that there is a labeling $\sigma_{V}$ to $V$ that satisfies $\zeta$ fraction of edges of $\mathcal{L}$. Consider the following randomized labeling $\sigma_{U}$ to $U$ : independently for each $u \in U$, choose a neighbor $v \in V$ uniformly at random, and assign $u$ the label $\pi^{e, v}\left(\sigma_{V}(v)\right)$. It is easy to see that the expected fraction edges of $\mathcal{B}$ satisfied by $\sigma_{V}$ and $\sigma_{U}$ is the probability over a uniformly random $u \in U$, and two of its neighbors, $v$ and $w$, chosen independently and uniformly at random, that $\pi_{v u}\left(\sigma_{V}(v)\right)=\pi_{w u}\left(\sigma_{V}(w)\right)$. This is exactly the probability over the choice of a random edge $e=\{v, w\}$ of $\mathcal{L}$, that $\pi^{e, v}\left(\sigma_{V}(v)\right)=\pi^{e, w}\left(\sigma_{V}(w)\right)$. By our assumption this is at least $\zeta$. Therefore, if $\mathcal{B}$ is a NO instance, then $\mathcal{L}$ is also a NO instance.

Finally, to see the weak expansion property, let $V^{\prime} \subseteq V$ such that $\left|V^{\prime}\right|=\delta|V|$. For each $u \in U$, let $p_{u}$ be the fraction of the neighbors of $u$ that are in $V^{\prime}$. Thus, $\mathbb{E}_{u}\left[p_{u}\right]=\delta$, and the fraction of edges ${ }^{1}$ in $\mathcal{L}$ induced by $V^{\prime}$ is $\mathbb{E}_{u}\left[p_{u}^{2}\right] \geqslant \mathbb{E}_{u}\left[p_{u}\right]^{2}=\delta^{2}$.

\section{REFERENCES}

N. Alon, K. Makarychev, Y. Makarychev, and A. Naor. 2006. Quadratic forms on graphs. Inventiones Mathematicae 163, 3 (2006), 499-522.

\footnotetext{
${ }^{1}$ We are also counting the (negligible) fraction self-loops produced in $\mathcal{L}$.
} 
N. Alon and A. Naor. 2006. Approximating the cut-norm via Ggrothendieck's inequality. SIAM J. Comput. 35, 4 (2006), 787-803.

S. Arora, B. Barak, and D. Steurer. 2010. Subexponential algorithms for unique games and related problems. In Proceedings of the 51st IEEE FOCS. 563-572.

S. Arora, E. Berger, E. Hazan, G. Kindler, and M. Safra. 2005. On non-approximability for quadratic programs. In Proceedings of the 46th IEEE FOCS. 206-215.

S. Arora, S. Khot, A. Kolla, D. Steurer, M. Tulsiani, and N. K. Vishnoi. 2008. Unique games on expanding constraint graphs are easy: Extended abstract. In Proceedings of the 40th ACM STOC. 21-28.

S. Arora, C. Lund, R. Motwani, M. Sudan, and M. Szegedy. 1998. Proof verification and the hardness of approximation problems. J. ACM 45, 3 (1998), 501-555.

S. Arora and S. Safra. 1998. Probabilistic checking of proofs: A new characterization of NP. J. ACM 45, 1 (1998), 70-122.

B. Barak, P. Raghavendra, and D. Steurer. 2011. Rounding semidefinite programming hierarchies via global correlation. In Proceedings of the 52nd IEEE FOCS. 472-481.

A. Brieden, P. Gritzman, and V. Klee. 2000. Inapproximability of some geometric and quadratic optimization problems. In Approximation and Complexity in Numerical Optimization: Continuous and Discrete Problems.

M. Charikar and A. Wirth. 2004. Maximizing quadratic programs: Extending Grothendieck's inequality. In Proceedings of the 45th IEEE FOCS. 54-60.

A. Deshpande, M. Tulsiani, and N. Vishnoi. 2011. Algorithms and hardness for subspace approximation. In Proceedings of SODA.

A. Deshpande and K. R. Varadarajan. 2007. Sampling-based dimension reduction for subspace approximation. In Proceedings of the 39th ACM STOC. 641-650.

D. Feldman, M. Monemizadeh, C. Sohler, and D. P. Woodruff. 2010. Coresets and sketches for high dimensional subspace approximation problems. In Proceedings of SODA. 630-649.

V. Feldman, V. Guruswami, P. Raghavendra, and Y. Wu. 2009. Agnostic learning of monomials by halfspaces is hard. In Proceedings of the 50th IEEE FOCS.

G. Golub and C. van Loan. 1996. Matrix Computations. Johns Hopkins University Press.

P. Gopalan, S. Khot, and R. Saket. 2010. Hardness of reconstructing multivariate polynomials over finite fields. SIAM J. Comput. 39, 6 (2010), 2598-2621.

V. Guruswami, J. Håstad, R. Manokaran, P. Raghavendra, and M. Charikar. 2011. Beating the random ordering is hard: Every ordering CSP is approximation resistant. SIAM J. Comput. 40, 3 (2011), 878914.

V. Guruswami and A. K. Sinop. 2011. Lasserre hierarchy, higher eigenvalues, and approximation schemes for graph partitioning and quadratic integer programming with PSD objectives. In Proceedings of the 52nd IEEE FOCS. 482-491.

S. Har-Peled and K. R. Varadarajan. 2002. Projective clustering in high dimensions using core-sets. In Symposium on Computational Geometry. 312-318.

S. Khot. 2002a. Hardness results for coloring 3-colorable 3-uniform hypergraphs. In Proceedings of the 43rd IEEE FOCS. 23-32.

S. Khot. 2002b. On the power of unique 2-prover 1-round games. In Proceedings of the 34th ACM STOC. 767-775.

S. Khot, G. Kindler, E. Mossel, and R. O'Donnell. 2007. Optimal inapproximability results for MAX-CUT and other 2-variable CSPs? SIAM J. Comput. 37, 1 (2007), 319-357.

S. Khot and O. Regev. 2008. Vertex cover might be hard to approximate to within 2-epsilon. J. Comput. Syst. Sci. 74, 3 (2008), 335-349.

S. Khot and R. Saket. 2006. A 3-query non-adaptive PCP with perfect completeness. In Proceedings of IEEE CCC. 159-169.

S. Khot and R. Saket. 2011. On the hardness of learning intersections of two halfspaces. J. Comput. Syst. Sci. 77, 1 (2011), 129-141.

G. Kindler, A. Naor, and G. Schechtman. 2010. The UGC hardness threshold of the $L_{p}$ Grothendieck problem. Math. Oper. Res. 35, 2 (2010), 267-283.

A. Megretski. 2001. Relaxations of quadratic programs in operator theory and system analysis. Systems, Approximation, Singular Integral Operators, and Related Topics, Vol.129. Springer Basel AG, 365-392.

E. Mossel. 2010. Gaussian bounds for noise correlation of functions. GAFA 19 (2010), 1713-1756.

A. Naor and G. Schechtman. 2010. An approximation scheme for quadratic form maximization on convex bodies. Preprint. 
A. Nemirovski, C. Roos, and T. Terlaky. 1999. On maximization of quadratic form over intersection of ellipsoids with common center. Math. Program. 86, 3, Ser. A (1999), 463-473.

Y. Nesetrov. 1998. Global quadratic optimization via conic relaxation. Working Paper, CORE (1998).

P. Raghavendra. 2008. Optimal algorithms and inapproximability results for every CSP? In Proceedings of the 40th ACM STOC. 245-254.

R. Raz. 1998. A parallel repetition theorem. SIAM J. Comput. 27, 3 (1998), 763-803.

N. D. Shyamalkumar and K. R. Varadarajan. 2007. Efficient subspace approximation algorithms. In Proceedings of SODA. 532-540.

K. R. Varadarajan, S. Venkatesh, Y. Ye, and J. Zhang. 2007. Approximating the radii of point sets. SIAM J. Comput. 36, 6 (2007), 1764-1776.

U. Zwick. 1998. Approximation algorithms for constraint satisfaction problems involving at most three variables per constraint. In Proceedings of SODA. 201-210.

Received February 2012; revised August 2015; accepted August 2015 